\title{
Functional RNA delivery targeted to dendritic cells by synthetic nanoparticles
}

Dendritic cells (DCs) are essential to many aspects of immune defense development and regulation. They provide important targets for prophylactic and therapeutic delivery. While protein delivery has had considerable success, RNA delivery is still expanding. Delivering RNA molecules for RNAi has shown particular success and there are reports on successful delivery of mRNA. Central, therein, is the application of cationic entities. Following endocytosis of the delivery vehicle for the RNA, cationic entities should promote vesicular membrane perturbation, facilitating cytosolic release. The present review explains the diversity of DC function in immune response development and control. Promotion of delivered RNA cytosolic release is discussed, relating to immunoprophylactic and therapeutic potential, and DC endocytic machinery is reviewed, showing how DC endocytic pathways influence the handling of internalized material. The potential advantages for application of replicating RNA are presented and discussed, in consideration of their value and development in the near future.

\section{Targeting the immune system}

Dendritic cells (DCs) play crucial roles in promoting and regulating immune defenses, providing important targets for prophylactic and therapeutic approaches (FIGURE I). Particulate formulations, including liposomes and other nanoparticles, have been employed as delivery vehicles. Despite the large number of reports, current in-depth knowledge on the cellular processes involved remains relatively limited, particularly for nucleic acid delivery. Certain structures in the nucleic acid may also signal the cell in the sense of an adjuvant or immunomodulatory activity. Importantly, the optimized characteristics for delivery of an antigen or therapeutic agent may be distinct from those for nucleic acid delivery, especially RNA species. Moreover, RNA delivery for interference therapy will not necessarily require the same delivery routes as mRNA delivery wherein RNA translation is the main requisite.

The efficacy of delivery can be further improved by targeting the appropriate cells of the immune system, an area in which nanoparticle-based delivery platforms have found an important niche [1]. Advances with prophylactic applications can also prove valuable for therapeutic applications and vice versa, as seen with RNA delivery.

This review will consider how growing knowledge on delivery mechanisms has found application with nucleic acids, in both prophylaxis and therapy, focusing on interaction with DCs.
The initial components of the DC endocytic pathways are critically important in determining how the cell handles the delivered material; thereafter, correct cytosolic delivery is a critical element for a number of desired outcomes, including delivery of nucleic acids. Advances made with protein delivery - in particular, vaccines - are of value to highlight the potential of a particular mode of application or the high risk for nucleic acid integrity. Particularly pertinent is the application of cationic elements in the delivery mechanisms and how application of ligands for cell receptors influence intracellular compartmentalization.

It is not the aim of the present review to retrace all the fine details of work contributing to our current knowledge. Accordingly, review articles covering areas that have already received considerable attention will be employed and assimilated to provide a more elaborate picture of the current situation. This will allow a focusing on more recent advances, along with problems and pitfalls therein. While advances on protein and DNA delivery will be presented, these will be used to highlight how our current knowledge can be applied to RNA delivery. There are numerous reviews on protein delivery to DC, wherein many of the procedures employed are not relevant for RNA delivery, which must escape into the cytosol undamaged. With DNA delivery, there is also the question of whether the DNA will activate the DC or reach the nucleus for transcription; this latter area has most often
Kenneth C McCullough*, Isabelle Bassi, Thomas Démoulins, Lisa J Thomann-Harwood \& Nicolas Ruggli

Institute of Virology \& Immunoprophylaxis, $\mathrm{CH}-3147$ Mittelhäusern, Switzerland *Author for correspondence:

Tel.: +4| 3 | 8489262

Fax: +4I 318489222

E-mail: kmc.projects।@gmail.com 


\section{Key Terms}

Dendritic cells: The critical cell to be targeted for promoting immune activation and immune regulation immunoprophylaxis and immunotherapy.

RNA delivery: Requires cytosolic release, and has high potential for RNAi therapy, as well as both therapeutic and prophylactic applications of translating RNA.

\section{Nanoparticle-based} delivery: The future for controlled targeting of dendritic cells, requiring careful consideration of their structure in terms of ligands to target dendritic cell receptors and cationic moieties to promote RNA release into the cytosol.

\section{Self-replicating RNA} replicon: Derived from replication-defective viral genomes; of high potential for future therapeutic and prophylactic applications; provide a higher output and more efficacious and long-lasting control (therapy) or stimulation (prophylaxis).

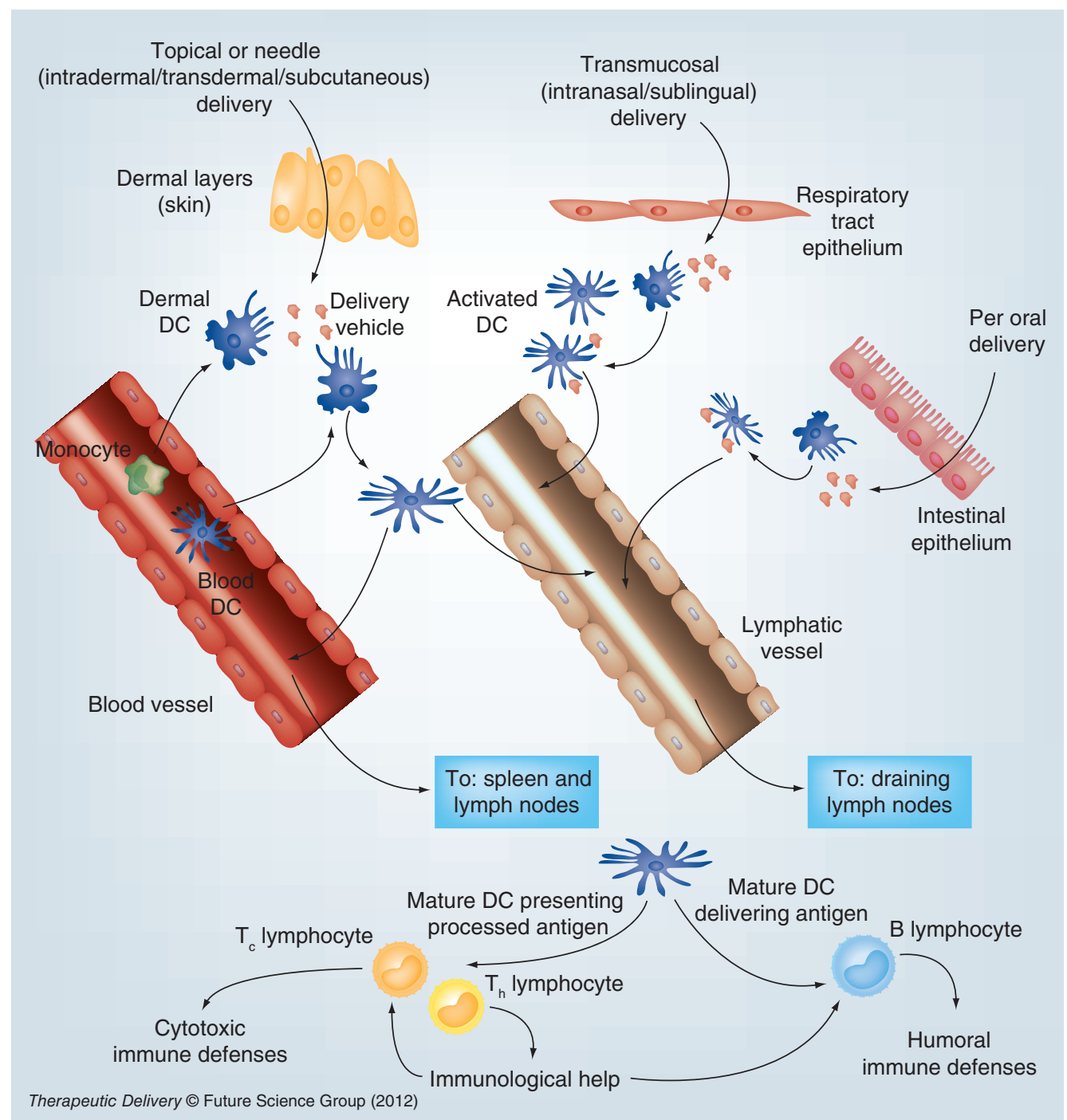

Figure 1. Dendritic cell subsets defined in terms of their site of encounter with delivered material in the body and their relationship to the secondary lymphoid organs, wherein they may deliver processed material for inducing immune responses. The trafficking operative following topical or needle delivery is compared with transmucosal delivery promoting translocation across the respiratory tract epithelium and per oral delivery promoting translocation across the intestinal epithelium. It should be noted that delivery via the mucosal surfaces, particularly the intestinal epithelium, can also promote tolerance (crosstolerance), dependent on the type of DCs with which it interacts, and the co-delivery of appropriate danger signals [30]. Translocation across the epithelial barrier of mucosal surfaces can be effected by transcytosis through a typical epithelia cell type, termed M-cells [115-117] or via direct interaction with protrusions from the DCs surveying the lumen of the tract [30,118-122].

DC: Dendritic cell.

been studied in cells and cell lines with a dividing nucleus, and is not necessarily pertinent to the situation found in DC. Accordingly, the present review will focus on information necessary to understand how RNA can be delivered successfully to DC. This will include a review of our knowledge pertinent to RNAi therapy, and the delivery of both $\mathrm{mRNA}$ and self-replicating RNA replicons.

\section{The value of targeting DCs}

Advances in targeting DCs have gained much from protein-based vaccines, inducing antibodydependent (humoral) and cell-mediated immune defenses - delivery of antigen to B-lymphocytes for antibody production, to major histocompatibility complex (MHC) class II-restricted T-helper (Th)-lymphocytes for essential immunological help, and to MHC class I-restricted 
cytotoxic $\mathrm{T}(\mathrm{Tc})$-lymphocytes for cell-mediated defenses [2-6] (Figures I \& 2). Thereby, DCs play critical roles, promoting immune defense development and also regulating immune activity and homeostasis (Figure 3). The following subsections will present an overview of our current knowledge in these areas of immune response activation and control. Being such a diverse and complex field, it is impossible to provide an in-depth review of each aspect. Indeed, there are already numerous excellent reviews covering the various topics therein, which are cited in the text of each subsection and to which the reader is encouraged to refer.

\section{Targeting DC}

Efficient targeting of DCs ensures appropriate activation and processing of the delivered material $[2,3]$. Particulate formulations enhance the targeting and delivery of both prophylactic vaccines and therapeutic drugs [7-10]. With vaccines, the delivery must consider both the antigenic component and the adjuvant formulation to ensure efficacious immune defense induction.

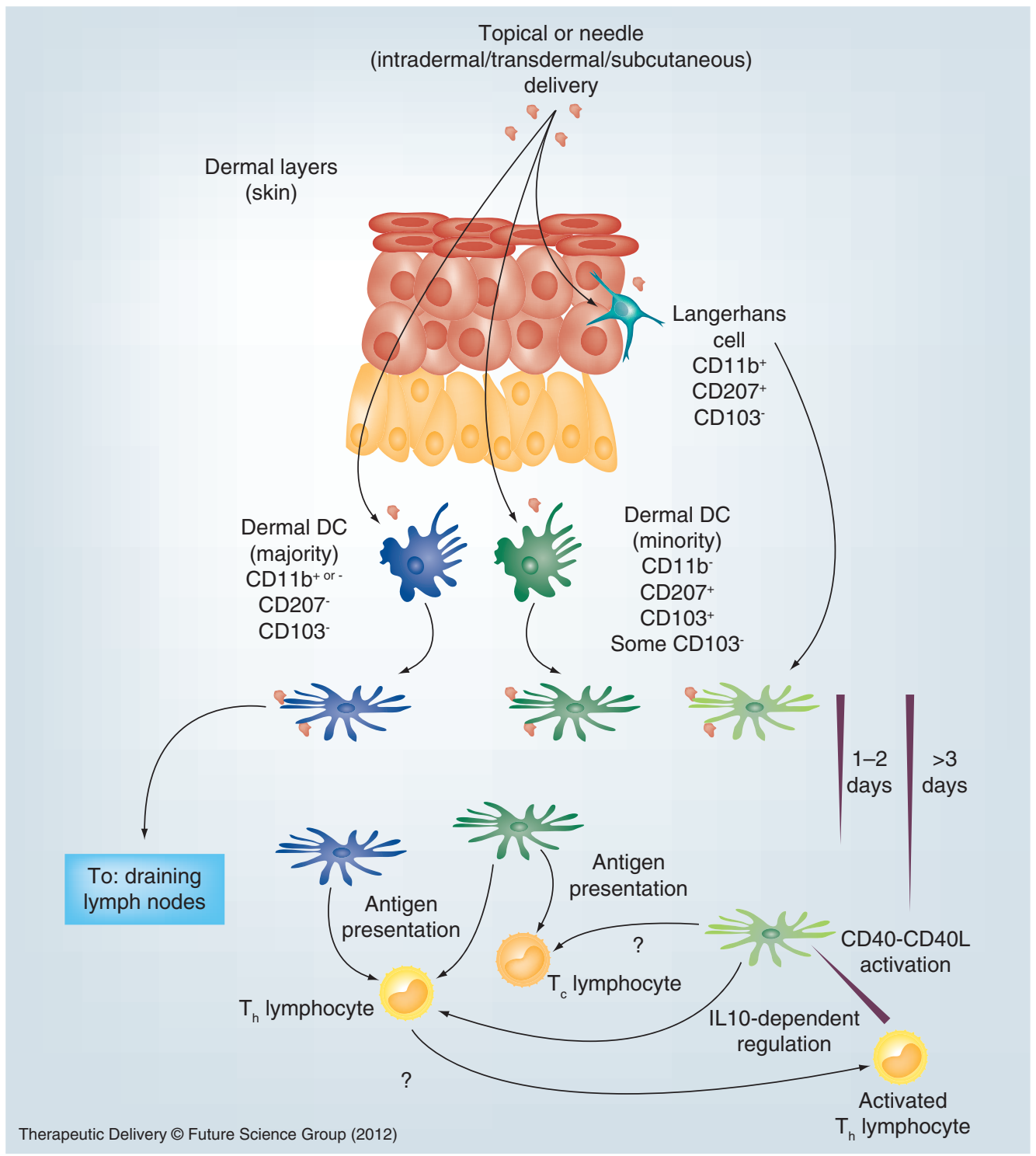

Figure 2. The importance of skin dendritic cell activity for interaction with delivery vehicles and communication with secondary lymphoid organs towards inducing adaptive immune responses. The different subsets of DCs in the dermal and epidermal layers, defined by their expression of CD11b, CD103 and Langerin (CD207), and how their interaction with a delivery vehicle results in variable rates of trafficking to draining lymph nodes resulting in different forms of immune response induction [47-50].

DC: Dendritic cell. 


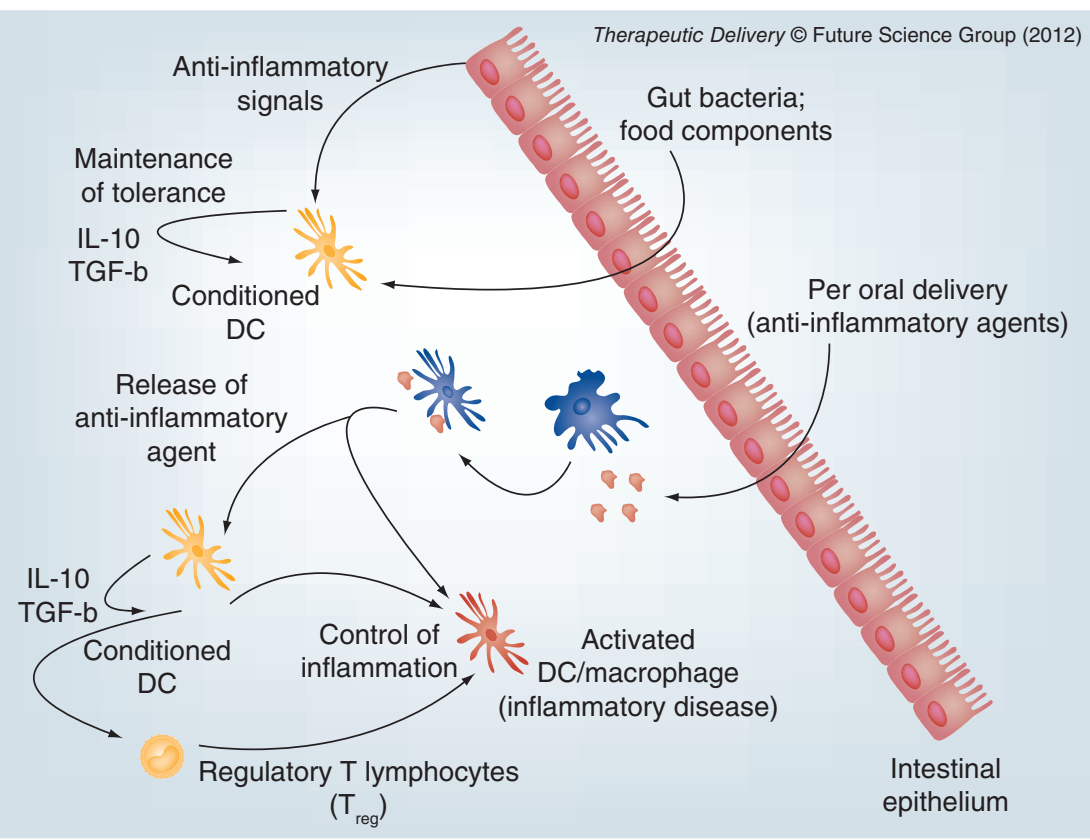

Figure 3. The particular roles played by dendritic cells at intestinal mucosal surfaces, with respect to controlling local tolerance and demonstrating how dendritic cell targeting can focus anti-inflammatory therapies in cases of intestinal inflammatory disorders. Mucosal immunity involves an interplay among several cell types, including the atypical epithelial cells referred to as $\mathrm{M}$-cells and the different DC subsets of the underlying intestinal lymphoid tissues, the specialized Peyer's Patches of the Lamina Propria [24,27,29-33] DC: Dendritic cell. endocytic process employed by DCs is macropinocytosis. Two other processes often reported with DCs are caveolar endocytosis and clathrindependent endocytosis. The latter route may be more important for degradation with most cell types, including DC, and is a major component of macrophage degradative pathways.

DC uptake of antigen for presentation to Th-lymphocytes

Although this area has been studied primarily with antigenic material endocytozed by DC, it is pertinent to RNA delivery. When RNA encodes for antigen, it has to be processed in a similar manner to endocytozed antigen; such RNA-derived material is transferred from its cytosolic site of translation into the endocytic pathways involved in processing antigens for presentation through MHC class II. Activating Th-lymphocyte function requires that DCs process endocytozed antigen or RNA-translated antigen into peptide fragments for associating with MHC class II molecules and presenting to the Th-lymphocytes. The efficiency and manner of processing is dependent on how the delivery vehicle carrying its cargo is endocytozed (Figure 4). An important consequence of endocytosis for processing and presenting to Th-lymphocytes is the interaction of the endocytic vesicle with the sorting endosomes $[2,5,17]$.

DC modify MHC class II molecules by oligoubiquitination of the cytoplasmic domain of the $\beta$-chain, driving the MHC class II molecules into sorting endosomes, thus, promoting 'sorting' with the endocytozed antigen. Acidification of the endocytic vesicles follows encounter with early endosomes, which provides the vacuolar $\mathrm{H}^{+}$-ATPases for acidification and the proteases (carboxypeptidases, aminopeptidases and endoproteases) for the processing. These proteases (termed cathepsins) are $\mathrm{pH}$ sensitive, hence the gradual acidification to $\mathrm{pH} 5.5$ as the structure matures from an 'early endosome' to a 'late endosome'. Endosomal maturation processes both the antigen and the MHC class II molecules to promote exchange of the antigenic peptides for the endosomally modified invariant chain (Ii) of MHC class II $[2,5,17]$. This occurs in the 'MHC class II-containing compartment' [2,5,17], from which MHC class II molecules loaded with antigen peptide are transported to the DC plasma membrane, for peptide presentation to Th-lymphocytes carrying T-cell receptors specific for the peptide being presented. 


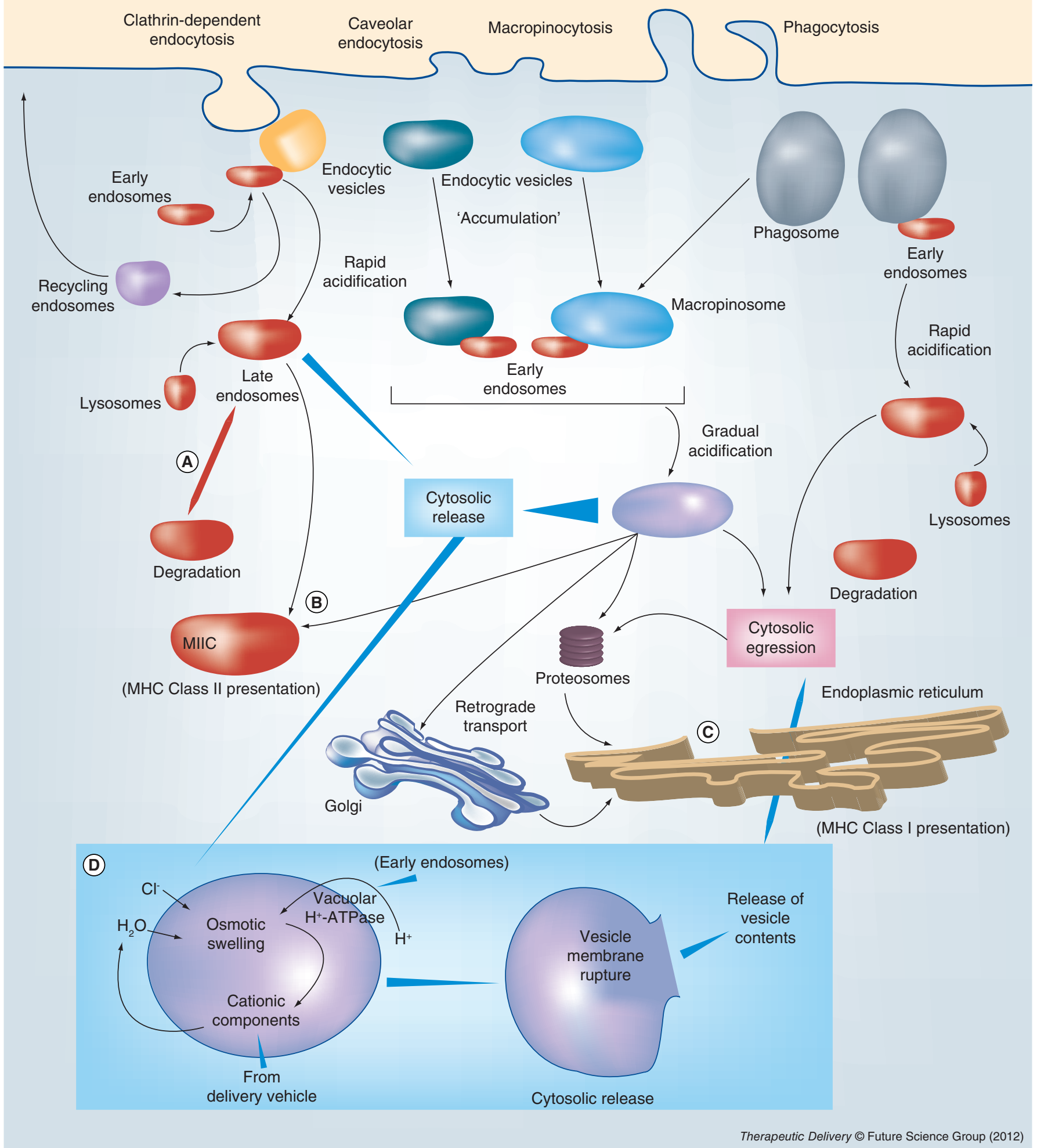

\section{Figure 4. How the different endocytic processes operative with dendritic cells can result in distinctive}

compartmentalizations. The figure relates to the potential outcome for delivered material in terms of antigen degradation or antigen processing for delivery to B-lymphocytes and presentation of peptides in association with MHC molecules to T-lymphocytes

[14-17,20-22,36,37,43,44]: (A) processes leading to degradation of the internalized material; (B) processing of the delivered material for MHC class II presentation (involving the MHC class II compartment or MIIC); (C) processing of the delivered material for MHC class I presentation. In (D), the cytosolic release important for cytosolic drug activities and RNA delivery leading to translation of mRNA and replicon RNA is represented $[80,81,84-86]$. 
Concomitant with antigenic peptide presentation is 'maturation' of the DC. While DCs endocytoze the delivered material in the periphery (dermal layers or mucosal surfaces, dependent on the route of application), they must migrate through lymphatics or the blood into draining lymph nodes or the spleen, wherein the responsive lymphocytes reside. Accordingly, the delivery must also activate the DC to upregulate chemokine receptors, such as CCR7, for promoting and directing migration into the lymph nodes. The maturation must also induce co-stimulatory molecule expression on the DC for the desired co-stimulation of the Th-lymphocytes, CD80 and CD86, to react with CD28 on Th-cells promoting activation or with CTLA-4 for regulation of activity. Application of adjuvants with vaccine formulations is one manner of ensuring appropriate maturation of the DC, through what is termed 'danger' recognition [11]. It is also possible to provide the danger signal alone, if activation of innate defenses is required in a therapeutic application.

\section{- DC processing antigen for priming or cross-priming}

DC also process antigens for presentation to Tc-lymphocytes, involving MHC class I molecules (Figure 4) $[2,4,6]$. The antigen can be generated within the cell, when it is referred to as endogenous antigen. Classically, this has been regarded as relating to antigens generated by infection of the cell with an intracellular parasite such as a virus. Importantly, when one considers nucleic acid-based vaccines, these too will translate their encoded proteins within the DC, thus providing an intracellular or endogenous source for processing. This is referred to as 'direct priming' of Tc-lymphocytes and was long thought to be the only mechanisms by which antigenspecific Tc-lymphocytes could be activated by $\mathrm{DC}$ antigen presentation in vivo [18]. As evidence accrued over the past decade, it became clear that an additional process to direct priming was available to DCs for presenting antigen via MHC class I processing [19].

Exogenous antigen, such as protein antigen endocytozed by the DC, can also be processed via the MHC class I pathways [18,19]. Such uptake of exogenous antigen is exemplified with conventional vaccines and those employing delivery vehicles for the antigen. DCs are also efficient at endocytozing dead and dying cells, which are a source of exogenous antigen. This may also occur when RNA is delivered if the
RNA induces death of the cell, as occurs with many of the current replicons under study as vaccines (see section titled 'Self-replicating RNA for prophylactic and therapeutic application'). The dying cells can be endocytozed by other DCs for further processing of the antigen they contain.

When DCs process exogenous antigen for MHC class I presentation, it is referred to as 'cross-presentation' [4,6,20-22]. Concomitant with appropriate DC activation and maturation events (see section titled 'DC processing antigen for tolerance, cross-tolerance or crosspriming'), cross-presentation leads to activation of the T-lymphocytes by 'cross-priming' [23-25]. These terms are designed to distinguish the DC activities from those associated with processing of endogenous antigen leading to priming $[18,19]$. Importantly, this may involve particular DC subsets, exemplified by the $\mathrm{CD} 8{ }^{+} \mathrm{DC}$ in the mouse, and be influenced by the size of material being endocytozed [4]. Indeed, the different DC subsets may process different types of antigen and require different signaling for activation. This is observed by the capacity of $\mathrm{CD}^{+}$ DC to constitutively cross-present antigen in immune complexes, while the CD8- DC require the assistance of activation via $\mathrm{Fc} \gamma$ receptor ligation [26].

\section{- Lessons from the controversy over cross-presentation \& cross-priming}

With the controversy over whether crosspresentation and cross-priming actually existed at the beginning of the new millennium, Norbury and Sigal deduced that there must be 'significant redundancy' between the two processes [18]. It is now clear that both priming and cross-priming (from cross-presentation) exist and that different DC subsets are involved [19]. Kurts et al. highlighted in their review why there had been controversy over cross-presentation for so long and exemplified the reasons why care must be taken on how in vitro analyses are designed and interpreted. The acceptance of cross-priming was clouded by the fact that viruses were often used in the studies; many viruses can infect DC, resulting in priming through endogenous antigen production, which would interfere with any capacity to observe cross-priming.

There were also problems with both the sensitivity of assays for measuring cross-presentation, resolved with improved techniques, and the current knowledge that certain epitopes may be too rapidly degraded for cross-presentation to be detected. Most importantly, the use of 
immortalized cell lines can create misleading results, because cell lines do not behave like primary cells; this is further complicated by the now known fact that particular DC subsets are involved in cross-presentation and crosspriming, while certain isolation procedures can damage DC receptors and their endocytic capacities. The authors determined that no DC-like or monocyte/macrophage-like cell lines carry the same receptor profile as primary DCs, and their capacity to endocytoze and process chitosanbased nanogel delivered cargoes is quite distinctive [McCullough K ET AL., Unpublished Data]. While much of this has been discovered in relation to cross-presentation, it is important for targeted delivery to DCs that one must pay attention to the DC subset under investigation and avoid over-interpretation of results with cell lines.

\section{- DC processing antigen for presentation to Tc-lymphocytes}

The main function of cross-presentation is to direct exogenous antigens into the same processing pathways for MHC class I presentation, as employed with endogenous antigens. Consequently, many of the intracellular events associated with cross-presentation are similar to those with processing of endogenous antigens for MHC class II presentation. Indeed, crosspresentation can utilize any one of the different endocytic processes employed by DCs with MHC class II processing [4,6,20-22]. Although antigens may be in similar vesicles as for MHC class II presentation, the processing for association with MHC class I molecules follows a less acidic course.

For MHC class I presentation, both endogenous (priming) and exogenous (cross-priming) antigen are processed internally by the cell using similar pathways; the differences lie in how the original antigen arrives into the MHC class I presentation pathways. Exogenous antigens must be released into the cytosol from the vesicular compartment into which it has been endocytozed by the DC. By these means, the cross-presentation pathway is initiated. Antigens thus released may be transferred into the endoplasmic reticulum (ER) by retrograde transport $[4,21]$, a process also found with the processing of endogenous antigen, as would be translated by delivered nucleic acid. Once the endocytozed exogenous antigens and the intracellular translated endogenous antigens are found in the cell cytosol, cross-presentation and endogenous antigen presentation pathways can follow similar routes. Another important characteristic of the endocytic pathways involved with exogenous antigen processing is the cytosolic release; this relates to the requirement of RNA for cytosolic release and we can learn much from the manner by which this release can be promoted.

With antigen presentation via MHC class I, the processing of antigens classically employs polyubiquination, targeting the material into the immunoproteasome, wherein the peptide antigens are generated. These are chaperoned by the transporter associated with antigen processing (TAP) back into the ER for association with MHC class I molecules, although the TAP transport can also deliver into an endosomal structure, wherein association with MHC class I molecules occurs [22]. A TAP-independent association with MHC class I may also arise, generation of the peptides and association with MHC class I molecules occurring in an endosomal compartment. Overall, the outcome is presentation of MHC class I-associated peptide antigens at the cell surface for interaction with the T-cell receptors of Tc-lymphocytes.

\section{- DC processing antigen for tolerance, cross-tolerance or cross-priming}

In addition to the above processes, one can focus delivery on excluding a danger signal in therapeutic approaches to promote DC processing of the antigen in the absence of co-stimulatory molecule expression. By such means, Th-lymphocyte and/or Tc-lymphocyte anergy or tolerance can be induced [27]. Indeed, it was thought that antigen presentation by immature DC, which do not express co-stimulatory molecules due to a lack of danger signals, was the primary manner for inducing tolerance. Self-reactive T-lymphocytes can be removed by negative selection through self-antigen presentation by thymic DC, but some self-reactive T-lymphocytes must be removed in the periphery for maintenance of self-tolerance [24]. For the latter, the migrating steady-state DCs are important for cross-presenting self antigens while in an immature state, inducing deletion or anergy in self-reactive Th- and Tc-lymphocytes. This is known as tolerance induction of autoreactive $\mathrm{CD}^{+}$Th-lymphocytes and 'cross-tolerance' with $\mathrm{CD}^{+}$Tc-lymphocytes [28]. It is beyond the scope of the present review to consider in detail the various aspects of tolerance, particularly the complexities involved with tolerance at mucosal surfaces. For more detailed information on this area, the reader is recommended to 
read the numerous reviews already published on tolerance [24,27,29-33]. The remainder of this subsection will highlight certain elements that are particularly pertinent when designing delivery to mucosal DCs for the induction of local immunity or tolerance (as in the case of an inflammatory disorder such as inflammatory bowel disease). It is important to note that, in both the periphery and at mucosal sites, the aforementioned incapacitation of the autoreactive T-lymphocytes is not always observable in terms of deletion of particular clones or induction of anergy in the cells. Another mode of tolerance induction is the conversion of these autoreactive T-lymphocytes into $\mathrm{CD}^{+}$regulatory T-lymphocytes [28]; the outcome is again determined by the interplay involving DC subsets, their activation state and the cytokine profiles in the environment (Figure 3).

It can be seen that cross-tolerance is a manifestation of cross-presentation under particular conditions of DC activation or responsiveness. When cross-presentation leads to activation of the T-lymphocytes via cross-priming [23-25], there are similarities with MHC Class II presentation in terms of danger recognition by the DCs (inducing upregulation of co-stimulatory molecules). Together with antigen presentation, the activation and maturation of DCs are important for defining activation or tolerization of T-lymphocytes [24]. However, this does not always hold true. An important additional requirement is a particular cytokine profile. For example, IL-12 is important for delivering activation signals, with IL-10 more important for the tolerance/anergy signaling (FIGURE 2). In fact, numerous environmental signals are important for regulating DC function and, therefore, determining the characteristics of the outcome arising from antigen presentation [34]. This led to the suggestion that mature DCs may also induce tolerance, but is now thought to depend on the interaction of DC, Th-lymphocytes and Tc-lymphocytes [24]. If Th1lymphocytes (IFN- $\gamma$ and IL-12 producers) are activated to induce DC expression of serine protease inhibitor-6, the DCs can block Tc-lymphocyte induction of apoptosis in the DC. IL-10 production by Th2-lymphocytes inhibits serine protease inhibitor- 6 expression by DC, rendering them susceptible to Tc-lymphocyte-induced death.

\section{- DC subsets in cross-presentation}

In addition to the influence of particular cytokine profiles in determining activation or tolerization events, particular DC subsets may play a more substantial role [19]. Certain subsets of dermal DC, Langerin ${ }^{+}, \mathrm{CD} 205^{+}$and $\mathrm{CD}_{103}{ }^{+}$, are similar to $\mathrm{CD} 8 \alpha^{+} \mathrm{DC}$ in promoting antigen cross-presentation (FIGURE 2); yet, inflammatory conditions can give rise to $\mathrm{CD} 103^{-}$and $\mathrm{CD} 8 \alpha^{-}$ DCs displaying cross-presentation capacities [19]. Moreover, it was reported that the CD8 $\alpha$ - DC, rather than the $\mathrm{CD} 8 \alpha^{+} \mathrm{DC}$, in the mouse gutassociated lymphoid tissues are important for cross-presentation leading to cross-tolerance, and may be important in food tolerance [35].

The roles played by DC subsets are influenced by the involvement of the T-lymphocytes [19]. Particular DC subsets process antigens for presentation to Th- or Tc-lymphocytes, but the activation of Tc-lymphocytes to become effector cells also requires help from the activated Th-lymphocytes. Moreover, such activated Th-lymphocytes can provide the required secondary signal in terms of CD40L-CD40 ligation to assist DCs when danger signaling is absent [19] (FIGure 2). The latter are referred to as 'licensed' DC; they show upregulated costimulatory molecules and efficient presentation of antigen to T-lymphocytes. TLR ligation can further enhance the DC activity, particularly for cross-priming [19].

The above information demonstrates that manipulation of both DC and T-lymphocyte activity can influence how an immune response will develop, in terms of an active immune defense or a tolerization of an unwanted process (Figure 3). Application of adjuvants for vaccine delivery will play a central role in such events and signaling DCs for danger recognition can also manipulate the situation. In addition, the manner of targeting materials to the DCs will play a critical role, as exemplified by the involvement of CLR. Targeting antigens to mannosebinding C-type lectins can direct processing into MHC class I- or class II-restricted pathways $[4,21,22]$. The majority of endocytozed material delivered via macropinosomes accumulates in lysosomes, probably for degradation or MHC class II processing [21]. Nonetheless, the relatively low degradation rate following macropinocytosis by DC can facilitate retrograde transport into the ER [21]. Macropinocytosis and caveolar uptake can deliver to the ER without interaction with early endosomes or shortly after acidification commences. A limiting feature may be the rate of acidification and endosomal degradation [36].Macrophages mostly degrade endocytozed material through rapid recruitment and activation of lysosomal proteases. DCs are 
slower at degrading internalized material and their endosomal $\mathrm{pH}$ can be less acidic than that of macrophages. Targeting to favor retention in early compartments can lead to both class I and II presentation [36,37], as well as cytosolic egression. The latter represents an essential step for nucleic acid delivery, which will be elaborated in more depth under the sections titled 'DC sensing via protons and cationic entities' and 'Targeting DCs for nucleic acid delivery'.

\section{- Nucleic acid delivery \& DC promotion of cross-presentation}

When one considers the role of nucleic acids targeted to the DC, the action will be seen as direct priming of T-lymphocytes if the nucleic acid translates its encoded proteins. This activity of the delivered nucleic acid will create an endogenous generation of antigen and is sought when attempting to deliver anRNA vaccine; this will be dealt with under the section 'Choosing the type of RNA for delivery to DC'. The situation with DNA is somewhat different, due to the difficulties that DNA has in translocating the nuclear membrane of DC. A nuclear targeting signal may prove useful and particular liposomal formulations may facilitate nuclear translocation of delivered DNA [38], but this was reported for bone marrow progenitors and the DC-like cells derived from them. Jilek et al. reviewed the literature on DNA delivery to DC, although they noted that if a DC-like cell does express translated proteins encoded by a delivered DNA, it is uncertain how this DNA translocated into the nucleus [39].

A major problem occurs when trying to interpret results with cell lines. Recent reports on successful delivery of DNA leading to translation have used a mouse DC-like cell line [40,41], which probably has no relevance to the situation with primary DC, as witnessed with the former controversy over cross-presentation [19]. Indeed, when using human and primate DC, Melhem $e t$ al. reported that even nucleofection with DNA gave poor results, contrasting with nucleofection of a leukemic cell line [42]. These authors also showed that RNA nucleofection of the DC did show efficient translation of the encoded proteins.

With DNA delivery, a more successful scenario is likely from targeting a replicating cell, in which the DNA can enter the nucleus upon cell division. For example, DNA transfection of epithelial cells can generate the mRNA needed for translation of the encoded proteins. When the latter are for vaccination purposes, they have to be endocytozed by DCs for antigen delivery and presentation. Such a process would be crosspriming, in contrast with the direct priming possible when RNA is targeted to DCs and released successfully into the its cytosol. Approaches involving DNA are, therefore, likely to be quite different to those with RNA, and their targeting and delivery requirements quite distinct.

Of course, RNA may also be delivered to cells other than DC, allowing for their translation in the targeted cell leading to cross-priming by DC. With this review focusing on RNA and DC, the emphasis will remain on how RNA molecules have been delivered directly to DCs for achieving the desired readouts. In these situations, the RNA will translate in the DC providing endogenous material for delivery to B-lymphocytes and direct processing for MHC class I and class II presentations.

\section{- DC delivering antigen to B-lymphocytes}

DC can also deliver antigen in a more intact form to B-lymphocytes in the lymph node and spleen follicles (FIGURE I) [43,44], because they readily accumulate antigen in macropinosomes in a more unprocessed form. A small GTPase (Rab27)-dependent process facilitates the release of antigen for interaction with the B-lymphocytes. Such an accumulation in macropinosomes followed by release to activate B-lymphocytes is of value for nucleic acids, although their release should favor the cytosol (Figure 4). Moreover, following translation of the nucleic acid encoded material, the DC can deliver this in a relatively unprocessed state to B-lymphocytes; antigens can activate the development of humoral immunity, while a therapeutic agent can control unwanted B-lymphocyte activities.

\section{Particular considerations for targeting DCs with nucleic acid delivery - RNA delivery to DC}

The value of RNA delivery is exemplified when considering prophylactic applications. Using protein delivery, the efficient induction of MHC class II-dependent responses are not always paralleled by MHC class I-dependent responses $[4,6,22,36,37]$. A replicating vaccine is more likely to induce the different arms of immune defense, being more closely related to the manner by which immune defenses develop in response to infection [1]. With the nuclear membrane of DCs being somewhat resistant to nucleic acid 


\section{Key Term}

\section{Prophylactic and} therapeutic applications:

The future should show the high potential of synthetic particle delivery of self-replicating RNA replicons. translocation [1], RNA vaccines offer particular value and RNA delivery for therapeutic purposes has also gained momentum $[45,46]$. A major requirement is cytosolic translocation prior to the maturing endosomal system, destroying the endocytozed nucleic acid. This will be elaborated in the sections titled 'DC sensing via protons and cationic entities' and 'Targeting DCs for nucleic acid delivery'.

\section{- DC subsets}

Many applications require targeting of conventional or classical DC $(\mathrm{cDC})$. These are the 'professional antigen presenting cells', efficiently presenting antigens to naive T-lymphocytes, and cross-presenting antigen and apoptotic cells. Yet there are several DC subsets, some of which show functional differences among species. In mice, both $\mathrm{cDC}$ and plasmacytoid DC (pDC) can respond to oligodeoxynucleotides via TLR9, but only pDC show this propensity in humans and pigs. DC subset activity will also vary dependent on their localization in the body, as exemplified by mucosal (lamina propria) DCs compared with dermal DC. Lamina propria DCs appear as $\mathrm{CD} 103^{+}$and CX3CR $1^{+}$ DCs. There are also Tip-DC (TNF and inducible NO synthetase producing DCs), CD11 ${ }^{\text {low }}$ TLR5+ DC and CD11b+ DC subsets. These latter three subsets, as well as $\mathrm{CD} 103^{+} \mathrm{DCs}$, are involved in different aspects of immune response regulation and promoting isotype switching to $\operatorname{IgA}$ for mucosal immunity [47]. Tolerogenic DCs are important for maintaining tolerance to commensal bacteria and food at mucosal surfaces, and ensuring non-responsiveness to self-antigens (Figure 3 ). It is important not to target DCs resulting in a reversal of tolerance, but therapeutic applications may gain from inducing a tolerogenic signal. Mucosal application may prove more valuable for inducing tolerogenic states, while parenteral application involving the dermal/epidermal layers may prove more valuable for activating immune defenses (Figure 2 \& 3).

Most dermal DCs are CD207-, but the $\mathrm{CD} 207^{+}$dermal DCs have the highest state of flux (FIgure 2). CD $207^{+}$dermal DCs are mostly $\mathrm{CD} 103^{+} \mathrm{CD} 11 \mathrm{~b}$, although $\mathrm{CD} 103{ }^{-\mathrm{CD}} 207^{+}$ dermal DCs and CD11b-CD207 dermal DCs exist; $\mathrm{CD}_{103^{+}} \mathrm{DCs}$ are also found at intestinal sites, but these are CD207- cells [48-50]. Upon activation, dermal DCs and Langerin/ CD207 ${ }^{+}$Langerhans cells (LCs) migrate to the T-lymphocyte areas of lymph nodes; the LC migration being slower than dermal DC migration. Antigen presentation to Th-lymphocytes probably involves $\mathrm{CD} 207^{+}$and CD207- DCs, the latter being more prominent. Targeting $\mathrm{CD} 207^{+}$ dermal DCs will promote cross-presentation of antigen, and $\mathrm{CD} 207^{+}$dermal DCs rather than LCs may promote contact hypersensitivity responses. LC-bearing antigens apparently require interaction with Th-lymphocytes through CD40-CD40L binding to become fully active. They may also be involved in more regulatory responses, through their secretion of IL-10 (Figures 2 \& 3).

Intradermal (percutaneous) delivery will reach the dermal DC, mostly bypassing the LC (Figures I \& 2). Transdermal (transcutaneous or epicutaneous) delivery increases the chance for interaction with LCs and dermal DC. Intranasal application will interact with mucosal DC, in the respiratory tract or the intestine if the delivery vehicle can resist the $\mathrm{pH}$ of the stomach. Interestingly, the slightly acidic $\mathrm{pH}$ of the upper respiratory tract and nasal passages may aid delivery by cationic delivery vehicles requiring a lowering of $\mathrm{pH}$ to release their cargo. This will be elaborated in the sections titled 'DC sensing via protons and cationic entities' and 'Targeting DCs for nucleic acid delivery'.

\section{DC sensing via receptors for targeted delivery \\ - DC receptors for recognizing 'danger'} Studying DC receptor targeting for prophylactic and therapeutic applications can use the same core procedures, whereby modifying the receptor ligand or applying the delivery vehicle at a different site - for example, mucosal surfaces rather than injection into the skin - can determine whether immune activation or regulation is the outcome (Figures $\mathbf{2} \& \mathbf{3}$ ). The $\mathrm{CDC}$ and $\mathrm{pDC}$ have distinctive arrays of PRR recognizing different PAMPs $[1,11,51]$. Cell surface PRR on cDC, such as receptors for lipoproteins (TLR2/TLR1 and TLR2/TLR6) and lipopolysaccharide (TLR4) [11] may present a potential for targeting. However, this may influence cell signaling, which is important considering the role of TLR signaling in regulating immune responses [51]. In this context, it is important to note that targeting DC receptors not involved in danger signaling may also prove applicable for efficient delivery to the cells. Targeting antigens to DCs in the absence of danger signaling may be used to induce regulation or tolerance, yet one may also employ PRR targeting with the same regulatory aim in mind. 
Inducing the desired manipulation of immune response development - activation or regulation - is not restricted to the DC surface PRRs. The cells also possess internal PRR recognizing nucleic acid-PAMP, such as the TLRs (TLR3, TLR7/8 and TLR9), nucleotide oligomerization domain-like receptors and cytosolic helicase sensors. They are valuable for intercellular activation, an approach already employed with PAMP-delivered therapeutic applications.

\section{- DC receptors for targeting}

DC possess a broad spectrum of cell-surface receptors in addition to TLR, with potential for targeting [1]. CLRs, Siglecs, galectins and scavenger receptors all offer this potential [11,51,52], particularly CLRs for their roles in endocytosis [9,53-56]. Many of these receptors are also found on macrophages, but DCs and macrophages do not posses an identical array of surface receptors. Moreover, their responses following ligation of the receptors is not necessarily identical.

Even when receptors found on both DCs and macrophages are targeted, such as mannosebinding receptors, the evidence from the literature shows that the DC can be successfully targeted. This question of the involvement of macrophages or DCs is a major point for vaccine delivery, yet vaccines that interact with both DCs and macrophages are successfully applied. Clearly, nature has designed the system to provide more degradative macrophages and slower processing DCs for antigen delivery and presentation [36]. Amigorena and Savina reviewed the work showing that macrophages recruit lysosomal proteases to phagosomes earlier and at higher levels than with DC; this related to DC degrading proteins at lower rates than with macrophages and having a phagosomal/endosomal $\mathrm{pH}$ less acidic than the counterparts in macrophages [36]. How the two cells operate in vivo is not fully understood, but there is certainly cooperation, further elaborated by the involvement of the inflammatory response in recruiting additional DC, macrophages, Th-lymphocytes and natural killer cells. It is possible that the macrophage is more important for the inflammatory response and, certainly, removal of any damaged or dying cells in the vicinity; the DC would be more involved in preparing antigens for activation of lymphocytes in secondary lymphoid organs. The activated DC certainly appears as the major player in migration to the lymphoid follicles for promoting the adaptive immune responses mediated by B- and T-lymphocytes.
Considering DC receptors for targeting, the family of mannose-recognizing CLR have been widely applied for vaccine and therapeutic delivery, even though such receptors are not necessarily unique to DCs. One has to determine the value of the targeting motif in terms of how different cells carrying the receptor will behave. This is particularly important when both DCs and macrophages are involved, wherein macrophages may be the more dominant. Targeted delivery may have to facilitate the DC interaction in a background of macrophage activity, whereby the influence of the macrophages on DC behavior and inducing an inflammatory response, would require elucidating.

With the skin being a common site for delivery of prophylactic and therapeutic agents, it is important to define the DC subset to be targeted (FIGURE 2) [53,56]. Ligands or antibody against the CLR CD205 (DEC-205) have been used, but CD205 is expressed on LCs as well as $\mathrm{CD} 207^{+}$and CD207- dermal DCs. Targeting Langerin (CD207) has also been attempted, but this will also target $\mathrm{CD} 207^{+}$dermal DCs. As mentioned above, $\mathrm{CD} 103{ }^{+} \mathrm{CD} 207^{+}$dermal DCs are important for cross-presentation, with CD103- CD207 dermal DCs more important for Th-lymphocytes activation. Application in vivo has targeted dermal DCs, or both dermal DCs and LCs [49]; LCs were noted to be superior to other skin DCs for inducing Tc-lymphocyte responses [49], promoting anti-tumor responses in vivo [57].

\section{DC sensing of protons \& cationic entities}

Delivering nucleic acids requires their cytosolic release from the endocytic vesicle, into which the delivery vehicles were internalized. Advances in cytosolic delivery of nucleic acids can benefit from successful approaches with certain proteins and lipoproteins which required cytosolic delivery. Two elements arise as being critical for promoting such delivery - sensing of protons and application of cationic entities.

\section{- Cationic lipid-based delivery to DC}

With the phospholipid bilayer of cell membranes presenting a strong barrier to ion transport, translocation of nucleic acids creates a challenge. Targeting RNA delivery to DCs has tended to employ ligands for DC receptors to assist that delivery [58,59], together with cationic lipids for facilitating cytosolic translocation. Liposomes have been widely applied for cationic-based 
delivery to DC, enhancing endocytosis of proteins and nucleic acids [60]. The anionic nature of the particular nucleic acid molecule dictates the applicability of cationic liposomes.

Cationic lipids with an amphiphilic nature can form micellar, lamellar, cubic and inverted hexagonal phases in aqueous environments such as biological fluids, the structure being dependent on the ratio of hydrophobic and hydrophilic regions [61]. When cationic lipids interact with nucleic acids, the lipoplexes can self-assemble into nanostructures, which require stabilization to prevent extensive particle clustering [61]. This is important considering that DCs behave differently towards smaller, compared with larger, particulate structures. The cationic particles may bind with the plasma membrane: when a small area encounters a high cationic charge from aggregated cationic particles, the membrane structure can be rigidified or weakened, leading to plasma membrane destabilization and potentially cell death. It is following endocytosis that cationic delivery has most potential. In the endocytic vesicles, cationic lipids may again interact with the anionic phospholipids of the vesicular membrane, but a particularly important event is the acidification by endosomes. The latter can facilitate membrane perturbation and cytosolic release of the contents. Controlling particle formation to focus membrane destabilization within the endocytic compartment has advantages for cytosolic delivery. One application has employed 'helper' lipids, such as cholesterol and dioleoylphosphatidylethanolamine (DOPE). Sugar-linked biosurfactants, such as dimethylaminoethane carbamoyl cholesterol/DOPE, have also found favor for stabilizations. By employing $\mathrm{pH}$-sensitive cationic lipids, such as dimethylaminoethane carbamoyl cholesterol, lipoplexes can promote membrane destabilization when endosomes begin acidifying the endocytic vesicles. PEGylation of lipids can also assist - this will stabilize particles at neutral $\mathrm{pH}$ and prevent clustering, but be removed in the acidifying endocytic vesicle to permit destabilization of the vesicular membrane due to the exposed cationic nature of the lipoplex.

The size, surface charge and degree of aggregation of lipoplexes, lipid-based nanoparticles and liposomes are dependent on particle composition $[62,63]$. Yet, they also offer potential for enhanced interaction with DCs using decoration with targeting ligands or antibodies against CLRs [62]. Activation of innate immune defenses is also possible with lipidic structures, through
TLR signaling as well as the complement cascade. Indeed, both activation and regulation can be promoted - liposomes encapsulating antigen for immune response induction are referred to as type I liposomes; polymer-coated liposomes designed to regulate immune recognition are referred to as type II. For example, liposomal bisphosphonates can inactivate and transiently deplete monocytes and macrophages. Liposomal aldronate is also immunosuppressive, inhibiting tissue macrophage activities, while phosphatidylserine (PS)-presenting liposomes can display an anti-inflammatory effect.

\section{- Cationic lipid-based particles for in vivo interaction with DC}

Cationic liposomes can be employed for drug or antigen delivery as well as nucleic acid delivery $[38,60-62,64]$. The lipid structure can determine the efficiency of delivery to DCs and the immunological readout in vivo. Incorporation of a disulfide bond into the structure may enhance release of a nucleic acid cargo from lipoplexes, while Gemini-surfactants, which carry two hydrophobic domains symmetrically linked through spacers with hydrophilic cationic head-group, efficiently deliver DNA and RNA. Nevertheless, other cationic lipids that do not display these properties have proven efficiency at delivering.

The administration route in vivo influences the outcome of cationic liposome delivery [64]. These authors reported on liposomes that enhanced uptake by DCs in vitro and in vivo following intranodal injection. With intradermal administration, there was no obvious advantage of the liposomal delivery, while liposomes were disadvantageous for transcutaneous and intranasal administration. Both intranodal and intradermal vaccinations promoted a more balanced Th1/Th2 response; this contrasted with transcutaneous and intranasal vaccination, which favored the Th2 response obtained with free material.

Local delivery of liposomes to the GI tract may show stability problems [7]. In contrast, the more solid matrix of lipid nanoparticles can increase stability, but success was still influenced by particle size: $<60 \mathrm{~nm}$ facilitated systemic bioavailablity and 100-200 nm favored tissue accumulation. With lipid nanoparticles adhering to the gut wall, leading to cargo release upon enzymatic cleavage, application of targeting ligands may not be so valuable as for liposomes. This is a good example of the care needed in designing 
delivery vehicles - it is important to determine how a targeting ligand would influence delivery in vivo, and not simply monitor interaction with cells in vitro.

\section{- Cationic peptides for delivery to DC}

In addition to lipids, cationic peptides have been employed for delivery, one of the earliest being the Tat-derived peptide from HIV [65]. Initial interaction with the cell membrane involved heparin-based structures, which may be attributed to the anionic nature of heparin. These were probably heparan sulfate proteoglycans, relating to how a number of viruses attach to cells prior to infection [66]. Since then, several arginine-rich peptides have been identified for translocation across cell membranes; a key role is played by guanidinium headgroups forming bidentate hydrogen bonds with sulfates and phosphates [65].

Cationic, arginine-rich peptides have been employed for cytosolic delivery of nucleic acids [65]. Following initial endocytosis, endosomes interact with the endocytic vesicles, providing the vacuolar- $\mathrm{H}^{+}$ATPase pump for accumulating protons in the vesicles. This is a critical step for cytosolic delivery, but the mechanism may be dependent on both the peptide and the cells employed [67]. Escape from the endocytic vesicle occurs as protons accumulate through action of the vacuolar- $\mathrm{H}^{+}$ATPase. The protons interact with the cationic peptide, leading to increase in vesicular osmotic pressure and destabilization of the vesicular membrane.

Cationic peptides can also interact at the plasma membrane, weakening or rigidifying the areas interacting with the peptides. While this may reduce membrane flexibility, it is likely to require a degree of amphipathicity. Nevertheless, there is the physiological relevance of using high concentrations of cationic peptides in vivo and whether such levels could be attained on cells being targeted. For endocytic uptake leading to cytosolic translocation, the lower concentrations of peptide required are more likely to be effective at promoting cytosolic translocation.

Cationic and amphipathic peptides have been used for nucleic acid delivery [68]. Lundin et al. found that only certain peptides promoted cytosolic delivery, but there was no correlation with the peptide being cationic or amphipathic [68]. Zaro and Shen did note that the length of different oligoarginine peptides could influence cellular uptake, but cytosolic translocation less so [69]. Tünnemann et al. reported that increasing lengths of peptides favored endocytic localization in vesicular structures, but a minor fraction was probably released into the cytosol - detected as a biological readout of the cargo [70]. With smaller cargo sizes, as well as increased cationic charge of the peptide, a slower endocytic uptake and more rapid translocation occurred. Amphipathic peptides with anionic components can also promote delivery into cells, as exemplified by Endo-Porter ${ }^{\mathrm{TM}}$ [71]. Its lipophilic face facilitates binding to the plasma membrane, leading to endocytosis of the peptide and material in its vicinity. Upon vesicular acidification, Endo-Porter converts into a polycationic form,but it is uncertain if this would promote cytosolic delivery of intact RNA. Endo-Porter requires a $\mathrm{pH}$ of 5-6 for this conversion, which relates to late endosomes and lysosomes wherein destruction of the RNA is more probable.

\section{- Other ionic- and $\mathrm{pH}$-responsive} structures for delivery to DC

Polyion complex micelles associating polymethacrylic acid- $b$-polyethylene oxide and poly-Llysine with antigenic peptide deliver through a $\mathrm{pH}$-sensitive and ion-dependent process [72]. However, these authors did not measure cytosolic delivery, and the $\mathrm{pH}$ of 5 required for dissociation is unlikely to be of value for RNA delivery. Polycationic, amphiphilic cyclodextran has been used for DNA delivery [73], although this work focused on epithelial cell lines rather than DC. Dierendonck et al.reviewed the work employing hydrogen bonded capsules assembled using poly(methacrylic acid) and degradable dextran sulfate/poly-L-arginine; reductionsensitive linkages were incorporated into the polymers, without introducing polycations [74]. Following cellular uptake, they localized to late endosomes and lysosomes. While highmolecular weight hydrophilic cargoes favored retention within acidifying vesicles, the presence of a lipophilic cargo promoted cytosolic release, but no information was reported for nucleic acid delivery.

Polyplexes can promote cytosolic delivery, but require careful choice of the cationic residues. Accordingly, pH-responsive entities - $\mathrm{pH}$-responsive core-shell gel nanoparticles have been generated by crosslinking hydrogels of a nontoxic $\mathrm{pH}$-insensitive hydrophilic shell surrounding a hydrophobic $\mathrm{pH}$-responsive core structure [75]. This approach promoted cytosolic delivery of cargoes ranging from virus 
particles to oligonucleotides [75,76]. Polyplex formation also employed cyclodextrin, polyethylenimine (PEI), protamine, atelocollagen and poly-L-lysine [77]. Including buffering and $\mathrm{pH}$-activated polymers or membrane-interacting peptides can facilitate cytosolic translocation. PEI has been employed for RNAi in vitro and in vivo (reviewed by Laga et al. [78]), with which a hydrophilic coat may provide a stealth mechanism. Considering that the cell must circumvent this stealth after internalization to permit translocation of the cargo, polyplexes with PEI and poly-L-lysine for the formation of homopolymers are showing potential, as well as statistical copolymers of amino or ammonio groups employed as polycations.

Cationic nanoparticles prepared using chitosan crosslinked by sodium tripolyphosphate (TPP) have delivered RNA for RNAi therapy [45]. This was strongly influenced by the chitosan form and molecular weight: chitosan hydrochloride was superior to chitosan glutamate, and $110 \mathrm{kDa}$ was superior to $270 \mathrm{kDa}$. Harwood et al. did not test their system with DCs, but the authors' own work has shown that the strong cationic nature of chitosantripolyphosphate nanoparticles can damage DC plasma membrane integrity [Harwood ET AL., NANOGEL VACCINES TARGETING DENDRITIC CELLS: CONTRIBUTIONS OF THE SURFACE DECORATION AND VACCINE CARGO ON Cell targeting AND ACtivation (2012), Submitted].

\section{RNA delivery to DC}

\section{- Endocytic uptake}

Studies on DC endocytosis of delivery vehicles has often observed clathrin-mediated uptake [61] (Figure 4). However, rapid internalization and acidification, as with clathrin-mediated endocytosis, may lead to a more degradative process. This has been observed with DC internalizing synthetic virus-like particles. Clathrinmediated uptake was only witnessed early; the accumulation of synthetic virus-like particles in the cells was related to macropinocytic and lipid raft-mediated responses [79]. Hassane et al. reviewed the evidence that cells may employ at least three pathways for endocytosis of cationic peptides - macropinocytosis, clathrin-mediated endocytosis and lipid raft-dependent endocytosis (Figure 4) [65]. Although all of these processes would acidify through the action of vacuolar $\mathrm{H}^{+}$-ATPases, one major difference among the routes is the rate at which this occurs. With clathrin-mediated endocytosis, this is more rapid, compared with macropinocytosis and lipid raft-mediated uptake. Moreover, other structures such as sorting endosomes can be involved, leading to a more prolonged accumulation of endocytozed material before interaction with acidifying endosomes (FIGURE 4). These are important characteristics of DC, displaying a more gradual processing following internalization and retention of the material for longer periods of time than other cells such as the more degradative macrophages.

\section{- Perturbation of DC endocytic vesicle membranes}

The mode of cytosolic delivery accomplished by cationic delivery vehicles is referred to as the 'proton sponge' or 'pH-buffering' effect (Figure 4) $[80,81]$. When using cationic cores, they demonstrate an added advantage of facilitating interaction with RNA and protection from RNAases. Cationic liposomes have been employed, encapsulating the nucleic acids, as well as condensing or adsorbing them on to their surfaces, to form polyplexes and lipoplexes $[60,80,82]$. Chitosan, PEI and cationic peptide-based structures have also been applied $[80,81,83]$.

When the early endosomes interact with endocytic vesicles, providing the vacuolar $\mathrm{H}^{+}$-ATPases for pumping protons into the vesicle, the proton sponge effect can be initiated (Figure 4). Chitosan and PEI contain protonable amines, behaving as buffering agents through which these groups readily accept protonation. Both molecules offer the 'proton sponge' effect and gene delivery can profit from combined chitosan-PEI hybrid delivery vehicles [83]. Amino and cationic groups are important for facilitating protonation towards cytosolic release from endocytic vesicles [84], while histidine- and arginine-rich molecules, as well as lipids with histidine residues as polar heads, initiate the proton sponge effect through protonation of imidazole rings $[80,81]$. These protonation events increase ion and water uptake into the vesicular lumen, increasing osmotic pressure within the vesicles (Figure 4). This leads to swelling of the vesicle and membrane disruption, allowing cytosolic release of the contents.

Certain histidine-and arginine-rich peptides and polymers assist cytosolic release through interaction with the anionic vesicular membrane [81]. If such peptides bind at the edges of membrane pores, they reduce internal membrane tension. Amphiphilic peptides, as in polyplexes and lipoplexes, can insert into the endocytic vesicle membrane, thinning chain length and creating 
internal membrane tension $[80,85]$. They are converted from an anionic hydrophilic coil to a hydrophobic $\alpha$-helix by protonation of glutamic acid, but its occurrence at $\mathrm{pH} 5-5.5$ raises doubts about applicability for RNA delivery.

Fusiogenic peptides, such as GALA, have also been employed for membrane destabilization. Operative at $\mathrm{pH}<6.0$, they have proven applicable for nucleic acid delivery and improving delivery by cationic liposomes and nanoparticles [81,85]. Indeed, combining entities showing promise for cytosolic release can prove beneficial.

Interaction of lipoplexes containing cationic lipids with endocytic vesicle membrane is strongly influenced by the membrane PS [61]. When PS becomes expressed on the inner vesicular membrane (as opposed to the outer membrane), it can interact with the lipoplexes by ionic pairing, promoting electrostatic interactions to neutralize charges, repel hydration and decrease membrane curvature. Conversion from a lamellar to a non-lamellar phase may also prove important for dissociation of the nucleic acid cargo from the lipoplexes. Critically important is the formation of tight intermembrane interactions between the vesicular membrane and the lipoplex surface.

PEG is often linked to lipid analogues for reducing the removal of lipoplexes by macrophages in vivo, but the PEG must be removed to facilitate cytosolic delivery [61]. A pH-sensitive PEG promotes dissociation from the lipoplexes as the endocytic vesicle acidifies, while addition of 'helper' lipids, such as DOPE and cholesterol, can enhance uptake by the cells and facilitate the $\mathrm{H}_{\text {II }}$ phase formation [86]. This latter approach disrupts endosomal membranes when in combination with anionic phospholipids, probably by promoting non-bilayer structures.

\section{Choosing the type of RNA for delivery to DCs}

There has also been growing interest in RNA delivery, a major advantage for which is the irrelevance of the nuclear membrane for successful delivery and translation of the RNA [87]. The sensitivity of RNA to RNAases is a major problem. It is essential that the ribosomal entry sites remain intact; even a low level of RNase activity can destroy this. Any nicks in the RNA by endonucleases will result in premature termination of translation, providing incomplete products. Such problems are not inherent to all forms of RNA. The dsRNA and siRNA or shRNA molecules used in RNAi therapy may prove more resistant to RNAases, in terms of their function; in fact, the generation of endogenous dsRNA molecules involved in RNAi processes, employs RNAse activities, which are paramount. These molecules do not require translation, but are required to interact with cellular mRNA to promote the degradation of the latter; even a low level of complementarity can be effective, although this may lead to translational arrest of the mRNA, rather than its degradation (see section titled 'RNAi').

\section{- RNA delivery to DCs}

Nucleic acid may be internalized by DC, but this is not regarded as particularly efficient [88]. Delivery of 'naked' RNA is a rare event in the scientific literature, which is dominated by employment of delivery vehicles for both prophylactic $[38,60,64,87,89,90]$ and therapeutic $[59,84,87,90]$ applications. As mentioned in the previous sections, cationic and amphiphilic delivery vehicles offer high potential for the cytosolic delivery important with RNA. An important aspect is the cationic lipid composition of the delivery vehicle. Basha et al. noted that from four ionizable cationic lipids, lipidnanoparticles based on 1,2-dilinoleyl-4-(2dimethylaminoethyl)-[1,3]-dioxolane were the most efficient for cytosolic delivery of siRNA in murine macrophages and DCs [91]. The amphipathic peptide Endo-Porter forms stable complexes with siRNA and was also successfully delivered to macrophages in glucan shells [92]. A cationic nona-D-arginine construct was applied together with a DC-targeting ligand to deliver siRNA for knockdown of dengue virus replication in macrophages and DCs [93]. Lipid-based nanoparticles modified with an octa-arginine peptide and the $\mathrm{pH}$-sensitive fusiogenic GALA peptide promoted cytosolic delivery of siRNA; this targeted mRNA encoding the SOCS1 protein, the synthesis of which was successfully knocked down in mouse DCs [94].

\section{- RNAi}

Much of the effort in the RNA delivery field has focused on RNAi. This subsection will highlight the active components of RNAi that have been successfully applied with particulatebased delivery to cells; for more detailed information on RNAi and the pathways involved, the reader is encouraged to read the reviews by Kong et al. [95] and Davidson and McCray [96]. Nevertheless, it is worthwhile to highlight the 
important components for understanding how RNAi therapy can be successful.

RNAi is a component of the cell regulatory machinery $[95,96]$ employing homologous dsRNA, which interacts with the mRNA being targeted, leading to translation-repression and/or degradation of the latter. There are two main forms of effector RNA molecules involved in RNAi - siRNA and miRNA. The dsRNA giving rise to these effector molecules can be endogenous - derived from nuclear dsRNA - or artificially generated as shRNA. In the cytosol, these dsRNA molecules associate with the Dicer complex (a dsRNA-specific ATP-dependent member of the RNAse III family), which processes the dsRNA into siRNA duplexes of 21-23 nt. Upon association with the AGO protein in the precursor RNAi-inducing silencing complex (pre-RISC), one RNA strand (the 'passenger strand') is removed. The now mature RISC containing the 'guide strand' of siRNA targets the homologous sequence in mRNA, transporting this to cytoplasmic processing bodies for degradation of the mRNA.

The other effector molecule of RNAi, miRNA, is derived from large primary sequences transcribed from cell DNA and processed by the RNAse III Drosha into hairpin RNA, of approximately 70 nt (pre-miRNA) $[95,96]$. This is exported to the cytoplasm where, akin to the processes associated with siRNA generation, the pre-miRNA is cleaved by the Dicer complex to form the 21-23 nt miRNA. There is a major distinction in the effector function for miRNA compared with siRNA. Evidence supports a role for miRNA in blocking mRNA translation - post-transcriptional gene silencing - rather than the cleavage of mRNA observed with siRNA. It is thought that this is due to imperfect complementary binding of the miRNA with the target mRNA; the evidence points to the miRNA suppressing initiation of mRNA translation leading to translation arrest and storage of the mRNA in $\mathrm{P}$ bodies (the cytoplasmic domains in which regulatory RNAs are found). However, these distinctive effector mechanisms of siRNA and miRNA are not absolute. There is evidence reporting miRNA-mRNA transport into cytoplasmic processing bodies for mRNA degradation, particularly when the complementarity of the miRNA for the mRNA is high [95,96]. Moreover, siRNA may also lead to translation inhibition rather than degradation of the mRNA when complementarity is low [95,96].
This is an important point because inhibition of mRNA translation may be reversible.

Exogenous dsRNA, shRNA or siRNA have been employed for RNAi applications, using cationic-based delivery vehicles $[58,62,77,80,95]$. The cationic lipids 1,2-dioleoyl-3-trimethylammoniumpropane (DOTAP) and $N$-(1-(2,3-dioleoyloxy) propyl)- $N, N, N$-trimethyl-ammoniummethyl sulfate are among a long list of potential candidates [62,97]. Application of $\mathrm{pH}$-responsive cores of crosslinked hydrogel delivery vehicles have been reported [76], as has polysaccharide-based delivery $[45,58,77,83,98]$. Chitosan-based nanoparticle delivery vehicles have also been successfully employed for delivering siRNA in vitro and in vivo [45,77,83,98]. Application of PEI in the delivery vehicle can enhance siRNA delivery [46], as can reducible histidine-nanoparticles [77,99]; the latter use $\mathrm{pH}$-sensing for providing a proton sponge effect. The versatility of $\mathrm{pH}$-responsive crosslinked hydrogel particles [76] was applied to siRNA delivery, and $\mathrm{pH}$-sensitive polyion complex micelles - poly-L-lysine together with a double hydrophilic block copolymer of polymethacrylic acid- $b$-polyethylene oxide successfully delivering siRNA to murine bone marrow-derived DCs $[72,100]$.

mRNA for gene \& gene-product delivery mRNA has high potential for delivering encoded proteins, but is limited by its sensitivity to RNAases. As with RNA for RNAi, mRNA delivery has employed delivery vehicles promoting cytosolic release from endocytic vesicles, cationic entities being at the forefront. Importantly, long polymers may enhance stability of the mRNA interaction, but decrease the release of the mRNA for translation. Mockey et al. employed histidylated lipopolylexes for successful delivery of a mRNA-based cancer vaccine [101]. They formulated PEGylated, histidylated poly-lysine together with L-histidine- $(N, N$ di- $n$-hexadecylamine) ethylamide to form the liposomes, into which the mRNA with a polyA tail was encapsulated. Markov et al. efficiently delivered mRNA into murine DC, using cationic liposomes formed from polycationic lipids mixed with DOPE in a 1:1 ratio [38]. The cationic lipids carried one or two cholesterol residues or long-chain hydrocarbon substituents linked with spermine. Delivery efficiency varied dependent on the lipid modification and the cell type, with liposomes carrying either the two cholesterol residues or the long-chain hydrocarbon being the most efficient in vivo. For the 
latter, DCs were pulsed in vitro with melanoma antigens prior to intravenous injection. It was not clear which immunological parameters were responsible for the greater efficacy of the liposomes with two cholesterol residues or the longchain hydrocarbon. Numerous cytokines and cytotoxic T-cell activity measured by the authors did not distinguish these liposomes from the one-cholesterol liposomes, which showed lower antimetastatic efficacy.

Lipid-based delivery of mRNA to DCs can be enhanced using mannosylated lipids and mannosylated/histidylated lipoplexes [102,103]. The liposome component of the lipoplexes was formed using $O, O$-dioleyl $-N-[3 \mathrm{~N}-(N-$ methylimidazolium iodide) propylene] phosphoramidate and $O, O$-dioleyl- $N$-histamine phosphoramidate, with $\beta$-D-mannopyranosyl$N$-dodecylhexadecanamide as the mannosylated component. RNA was mixed with PEGylated, histdylated poly-L-lysine, then interacted with the liposomes to form the lipopolyplexes. Another successful modification was to coat $\mathrm{pH}$-responsive polymer particles with a lipid envelope [104], but this approach adsorbed the mRNA to the surface of the particles, which could prove problematic due to the sensitivity of mRNA to RNAases. Improved RNA stability, together with enhanced transfection efficiency and reduced toxicity, was observed with copolymer blends of PEI and PEI-PEG and cationic lipids DOTAP and DOPE [105,106], although no DC targeting was reported.

\section{Self-replicating RNA for prophylactic \&} therapeutic application

Non-replicating vaccines provide a limited number of molecules, resulting in a restricted number of immunostimulation rounds, and a direct impact on induced immune defense avidity and efficacy. Nucleic acid vaccines can provide several rounds of protein production in the cells with which they interact, thus, increasing the degree of immunostimulation and strength of the response. While mRNA can provide several rounds of translation and, therefore, more protein for a lower cargo delivery than if the antigen itself were applied, there is still a limit to the amount, which can be translated. Selfreplicating RNA can generate a larger number of mRNA templates than could easily be delivered and guaranteed to arrive in the correct cytosolic localization of the targeted cell, such as the DC. Similar advantages would be forthcoming with delivery of encoded therapeutic proteins.
Such self-replicating RNA are referred to as replicons. They are replication-competent, defective virus genomes. The most frequently applied replicons have used RNA genomes [107-110], such as those of alphaviruses, flaviviruses and pestiviruses. Being 'positive strand' viruses, their genomes function as both mRNA and templates for replication. This contrasts with 'negative strand' viruses, which must be delivered with their own polymerase to promote the initial replication and formation of mRNA templates. Delivery of the latter is more reliant on the use of virus-like particles, and not so pertinent to synthetic particle-based delivery.

Replicons efficiently replicate and translate, but cannot form infectious virus due to a lack of viral structural protein genes. They can be packaged into virus-like particles, called virus replicon particles (VRP), using complementing cell lines stably expressing the missing viral genes in a transducible form. VRP can be used to infect the same types of cells susceptible to infection by the virus, but the replicon can only replicate and translate in those initially infected cells; there is no extension into other cells or generation of infectious progeny. The replicative nature of replicons mimics more closely that in a natural virus infection, and will be more efficient at inducing both humoral and cytotoxic cellular immune defense arms.

The therapeutic and prophylactic potential of replicons has been reviewed by Rayner $e t a l$. [109] and Atkins et al. [110]. Most replicons are delivered as VRP or transfected; there are as yet no reports on delivery by synthetic particles. Racanelli et al. [111] developed a replicon from bovine viral diarrhoea virus, into which the NS3 of hepatitis $C$ virus was inserted near the 5' end after the internal ribosomal entry site. Following transfection of a DC line, this replicon efficiently induced cytotoxic immunity. Frey et al. [112] and Suter et al. [113] employed VRP based on the classical swine fever virus genome, demonstrating that a single inoculation was efficient at inducing both humoral and cellular immunity. Flavivirus replicons, such as those derived from West Nile fever virus, have been modified to deliver genes of dengue virus type 2 [114]. Again, a single inoculation was sufficient to induce anti-dengue-virus neutralizing antibodies and the animals showed increased resistance to challenge infection. Despite the prolonged survival, the animals did eventually succumb to the infection, demonstrating that application of replicon technology is not without its problems of efficacy. 
The replicon area is clearly in a stage of evolution, but remains reliant on the use of complementing cell lines to generate the VRP. Not only is this cumbersome and expensive, but it is restricted to the cell-targeting capacity of the virus, from which the replicon was derived, both species and cell-type restrictions. It certainly can employ the evolutionary processes that the virus developed for cytosolic escape. Therefore, the advantages offered by cationic delivery vehicles may prove particularly valuable for replicon delivery without the need for VRP generation. This is even more applicable when the replicon is generated to lack all viral structural genes, the complementation of which would be more difficult to achieve, and perhaps prohibitively expensive for vaccine or therapeutic application. The value of cationic lipids, peptides and particles is likely prove of high value in this area, but their promise will be dependent upon the manner by which the delivery vehicle is administered to the host and targeted to the DC.

\section{Conclusion}

Delivery of both proteins and nucleic acids to cells has made considerable advances in the past 10 to 15 years, most recently in the field of RNA delivery. Considering delivery to DC, both promotion of immune defense development and immunotherapy have been studied. With DNA, which must enter the nucleus, the resistance of the DC nuclear membrane does not favor this unless a nuclear targeting ploy is employed. In contrast, RNA is not restricted by having to cross the nuclear membrane; delivery must promote cytosolic translocation of the RNA from within the endocytic vesicles into which it was delivered. Therefore, processes for delivery into the cytosol are crucial mechanisms for aiding RNA delivery. An important aspect of this escape from the endocytic vesicle is the timing before endosomal maturation has led to degradation of the delivered cargo. With RNA being so sensitive to RNase activity, this is a most critical step. Moreover, the delivery vehicle must be capable of protecting the RNA from RNAases prior to uptake by the cells.

Studies on cytosolic delivery of proteins and peptides, either for antigen cross-presentation or for therapeutic application, have assisted in the development of procedures for cytosolic delivery of RNA. Therein, the application of amphiphilic and cationic entities in the delivery vehicle or delivery process have shown the importance of destabilizing the endocytic vesicle; this can be in terms of destabilizing the membrane structure or increasing the osmotic pressure within the endocytic vesicles. In both cases, the vesicular membrane is weakened and, ultimately, disrupts to release the vesicular contents into the cytosol. Cationic lipids and peptides, as well as cationic entities with amine groups or imidazole rings, are of high value in this area, acting as proton sponges when the endosomal system attempts to acidify the endocytic vesicles. This upsets the ion balance within the lumen of the vesicle, increasing water uptake and, therefore, osmotic pressure in the vesicle. The outcome is disruption of the endocytic vesicle and release of the endocytozed RNA into the cytosol.

When the RNA is double stranded and delivered for RNAi, most often as siRNA, this can ultimately interact with the RISC, to promote degradation of the mRNA, for which it is specific. Should the effector RNA molecules lack complementarity for the mRNA of interest in the therapy, translation arrest may occur, but this may prove to be reversible. In contrast with RNAi, delivery of RNA for translation seeks interaction of the delivered RNA with the ribosomal machinery. Such approaches aim to promote translation of the encoded gene product, which can prove a useful tactic for prophylactic or therapeutic application.

While cationic particle delivery of mRNA has also been reported, there is no information on replicon RNA delivery by synthetic particles as of yet. This is an important area, offering advantages over mRNA in terms of less material being required for the delivery. Importantly, the replicative nature of the replicon RNA increases the number of templates. This allows for increase in the amount of translated product, beyond that which could be delivered via mRNA or the more conventional protein-based and inactivated pathogen vaccines. With the advances in efficient cytosolic delivery of mRNA, particularly to DC, it is likely that similar synthetic approaches to the delivery of replicating replicon RNA will be forthcoming in the very near future.

\section{Future perspective}

Targeting RNA for RNAi therapy is already well advanced, particularly in the context of cancer therapy, although such approaches do not necessarily require DC. Such an approach involving DCs would become of value when dealing with immunological disorders. An example is with immunopathological disorders involving DC, particularly the $\mathrm{pDC}$ and its high potential for 
IFN- $\alpha$ production; such situations can be seen with inflammatory virus infections. Of course, there are a number of causes for excessive activity from DCs or macrophages, as with inflammatory bowel disease and inflammatory arthritis, which would benefit from RNAi-based therapy targeted to the DCs and/or the macrophages involved. With inflammatory arthritis, the excessive production of TNF- $\alpha$ could be countered by RNAi therapy. It is also conceivable that RNAi could be employed to fight virus infections, but the main approach with the majority of virus infections is vaccination. Syntheticbased particle delivery has proven successful for protein antigen and adjuvant delivery, and the potential for mRNA vaccine delivery has been identified. As with proteins and conventional inactivated pathogen vaccines, mRNA vaccines are limited in the amount of material they can provide, which, in turn, limits the strength and longevity of any induced immune response. Selfreplicating RNA replicon vaccines can resolve these problems and limitations. Being synthetic vaccines, they are not encumbered by delays and risks associated with current, more traditional vaccine production methods. Their replicative characteristics provide several rounds of antigen production, inducing long-lasting immunity. Moreover, this replication mimics that of a virus infection, thus being more likely to induce both humoral and cell-mediated immunity, compared with more traditional non-replicating vaccines.

\section{Executive summary}

\section{The value of targeting dendritic cells}

- Pathogen-associated molecular patterns are detected by particular dendritic cell (DC) receptors, leading to DC activation, but may prove of value for targeting to the DC.

- DCs are central players in processing antigens for presentation to the helper T-lymphocytes, thus providing essential immunological help for development of the various lymphocyte-based immune defenses.

- DCs process antigens for cross-presentation and cross-priming, but are also the main players in direct priming of cytotoxic T-lymphocytes.

- RNA delivered correctly to DC translates to provide endogenous antigens for direct priming of cytotoxic T-lymphocytes and presentation to helper T-lymphocytes.

- DC activities combined with interactions with other cells and factors in their local tissue environment determine the outcome of immune activation or tolerance.

- DCs also deliver antigens to B-lymphocytes.

Particular considerations for targeting DC with nucleic acid delivery

- Different DC subsets determine the characteristics of an immune response and, therefore, the outcome of RNA delivery.

\section{DC sensing via receptors for targeted delivery}

- The crucial role of 'danger' recognition by the DC determines immune activation or tolerance induction.

- Immune activation or tolerance induction is also reliant on the interactions of DCs with other cells and factors in their local tissue environment.

- Particular DC receptors can be used successfully for targeting, despite the occurrence of some, but not all, receptors on both DC and macrophages.

\section{DC cell sensing of protons \& cationic entities}

- Cationic lipids show a high value for successful cytosolic delivery to DCs.

- Cationic lipid-based particles enhance cytosolic release from the endosomal system, including in the DC.

- Cationic peptides are proving advantageous for cytosolic delivery, but may be faulted by their low pH requirements reducing their value for cytosolic release of RNA.

- Other ionic- and pH-responsive structures have been tested for delivery to DCs.

\section{RNA delivery to $D C S$}

- The endocytic route employed for uptake by DCs will define the likelihood of success for RNA delivery.

- Perturbation of DC endocytic vesicle membranes early following initial acidification of endocytic vesicles is of critical importance for successful cytosolic release.

\section{Choosing the type of RNA for delivery to dendritic cells}

- RNA has been delivered for RNAi therapy.

- mRNA has been successfully employed for gene and gene-product delivery.

- Self-replicating RNA has high potential for prophylactic and therapeutic application 
Targeting DCs will enhance the efficacy of such replicating vaccines, for which purpose the cationic delivery vehicles presented in this review are major candidates. Current work is defining the delivery vehicle characteristic required for efficient cytosolic delivery of the replicon RNA, leading to its translation and efficacious activation of both humoral and cell-mediated immune defenses. Unfortunately, there remains a lack of funding for such approaches, which is delaying advances in proof-of-concept development. Extensive preclinical and clinical studies will also be required before replicon vaccines delivered by cationic delivery vehicles targeting DCs see the market place. This has the potential to delay their application in society for another decade or more. Nevertheless, the application of self-replicating replicon RNA vaccines in synthetic delivery vehicles targeting the DC offers major advantages for promoting efficacious and long-lasting immune defense development. Moreover, these replicons can encode therapeutic compounds, which could also prove valuable in the future. To date, there have been no reports of advances in this area, but this is probably just a matter of time. Overall, the future perspective for self-replicating replicon RNA delivery to DCs holds much promise for prophylactic or therapeutic applications, particularly when applied with synthetic, cationic delivery vehicles.

\section{Financial \& competing interests disclosure}

The preparation of this article and the work of the authors mentioned therein was supported financially by the Institute of Virology and Immunoprophylaxis, and the grants PANFLUVAC (EU FPG project 044115), Replixcel (EU FP7, Marie-Curie Industry-Academia Partnerships and Pathways project 251420), HCVAX (EU FP7 ERANET Euronanomed and Swiss National Science Foundation project31NM30-136034) and NANOVACC (Swiss National Science Foundation project 310000-1198828). KC McCullough and N Ruggli are associated with the replicon technology for the swine fever virus replicon covered by patent numbers WO2009/146867, EP2130912 and WO2010/067318. The authors have no other relevant affiliations or financial involvement with any organization or entity with a financial interest in or financial conflict with the subject matter or materials discussed in the manuscript apart from those disclosed.

No writing assistance was utilized in the production of this manuscript.

\section{References}

1 McCullough KC, Summerfield A. Targeting the porcine immune system - particulate vaccines in the 21st century. Dev. Comp. Immunol. 33, 394-409 (2009).

2 Thery C, Amigorena S. The cell biology of antigen presentation in dendritic cells. Curr. Opin. Immunol. 13, 45-51 (2001).

3 Randolph GJ, Jacubzick C, Qu C. Antigen presentation by monocytes and monocytederived cells. Curr. Opin. Immunol. 20, 52-60 (2008).

4 Jensen PE. Recent advances in antigen processing and presentation. Nat. Immunol. 8, 1041-1048 (2007)

5 Rocha N, Neefjes J. MHC class II molecules on the move for successful antigen presentation. EMBO J. 27, 1-5 (2008).

6 Vyas JM, Van der Veen AG, Ploegh HL. The known unknowns of antigen processing and presentation. Nat. Rev. Immunol. 8, 607-618 (2008).

7 Collnot E-M, Ali H, Lehr C-M. Nano- and microparticulate drug carriers for targeting of the inflamed intestinal mucosa. J. Control. Release 161, 235-246 (2012).

8 Danhier F, Ansorena E, Silva JM, Coco R, Le Breton A, Preat V. PLGA-based nanoparticles: an overview of biomedical applications. J. Control. Release 161, 505-522 (2012).

9 Hamdy S, Haddadi A, Hung RW, Lavasanifar A. Targeting dendritic cells with nanoparticulate PLGA cancer vaccine formulations. Adv. Drug. Del. Rev. 63, 943-955 (2011).

10 Klippstein R, Pozo D. Nanotechnology-based manipulation of dendritic cells for enhanced immunotherapy strategies. Nanomedicine 6, 523-529 (2010).

11 Pulendran B, Ahmed R. Translating innate immunity into immunological memory: implications for vaccine development. Cell 124, 849-863 (2006).

12 Shortman K, Lahoud MH, Caminschi I. Improving vaccines by targeting antigens to dendritic cells. Exp. Mol. Med. 41, 61-66 (2009).

13 Unger WW, van Beelen AJ, Bruijns SC et al. Glycan-modified liposomes boost CD4(+) and CD8(+) T-cell responses by targeting DC-SIGN on dendritic cells. J. Control. Release 160, 88-95 (2012).

14 Kumari S, Mg S, Mayor S. Endocytosis unplugged: multiple ways to enter the cell. Cell Res. 20, 256-275 (2010).

15 Sandvig K, Pust S, Skotland T, van Deurs B. Clathrin-independent endocytosis: mechanisms and function. Curr. Opin. Cell Biol. 23, 413-420 (2011).
16 Platta HW, Stenmark H. Endocytosis and signaling. Curr. Opin. Cell Biol. 23, 393-403 (2011).

17 van Niel G, Wubbolts R, Stoorvogel W. Endosomal sorting of MHC class II determines antigen presentation by dendritic cells. Curr. Opin. Cell Biol. 20, 437-444 (2008).

18 Norbury CC, Sigal LJ. Cross priming or direct priming: is that really the question? Curr. Opin. Immunol. 15, 82-88 (2003).

19 Kurts C, Robinson BW, Knolle PA. Cross-priming in health and disease. Nat. Rev. Immunol. 10, 403-414 (2010).

20 Heath W, Belz G, Behrens GMN et al. Cross-presentation, dendritic cell subsets, and the generation of immunity to cellular antigens. Immunol. Rev. 199, 9-26 (2004).

21 Monu N, Trombetta ES. Cross-talk between the endocytic pathway and the endoplasmic reticulum in cross-presentationby MHC class I molecules. Curr. Opin. Immunol. 19, 66-72 (2007).

22 Burgdorf S, Kurts C. Endocytosis mechanisms and the cell biology of antigen. Curr. Opin. Immunol. 20, 89-95 (2008).

23 Bevan MJ. Cross-priming. Nat. Immunol. 7, 363-365 (2006). 
24 Blankenstein T, Schuler T. Cross-priming versus cross-tolerance: are two signals enough? Trends Immunol. 23, 171-173 (2002).

25 Melief CJ. Mini-review: regulation of cytotoxic T-lymphocyte responses by dendritic cells: peaceful coexistence of cross-priming and direct priming? Eur. J. Immunol. 33, 2645-2654 (2003).

26 den Haan JM, Bevan MJ. Constitutive versus activation-dependent cross-presentation of immune complexes by CD8(+) and CD8(-) dendritic cells in vivo. J. Exp. Med. 196, 817-827 (2002).

27 Moser M. Dendritic cells in immunity and tolerance-do they display opposite functions? Immunity. 19, 5-8 (2003).

28 Lutz MB, Kurts C. Induction of peripheral CD4 + T-cell tolerance and CD8 + T-cell cross-tolerance by dendritic cells. Eur. J. Immunol. 39, 2325-2330 (2009).

29 Allam JP, Bieber T, Novak N. Dendritic cells as potential targets for mucosal immunotherapy. Curr. Opin. Allergy Clin. Immunol. 9, 554-557 (2009).

30 Rescigno M. Dendritic cells in oral tolerance in the gut. Cell. Microbiol. 13, 1312-1318 (2011).

31 Rescigno M, Di Sabatino A. Dendritic cells in intestinal homeostasis and disease. J. Clin. Invest. 119, 2441-2450 (2009).

32 Rescigno M, Lopatin U, Chieppa M. Interactions among dendritic cells, macrophages, and epithelial cells in the gut: implications for immune tolerance. Curr. Opin. Immunol. 20, 669-675 (2008).

33 Rescigno M, Matteoli G. Lamina propria dendritic cells: for whom the bell TOLLs? Eur. J. Immunol. 38, 1483-1486 (2008).

34 Sabatte J, Maggini J, Nahmod K et al. Interplay of pathogens, cytokines and other stress signals in the regulation of dendritic cell function. Cytokine Growth Factor Rev. 18, 5-17 (2007).

35 Chung Y, Chang JH, Kweon MN, Rennert PD, Kang CY. CD $8 \alpha-11 b+$ dendritic cells but not CD $8 \alpha+$ dendritic cells mediate crosstolerance toward intestinal antigens. Blood 106, 201-206 (2005)

36 Amigorena S, Savina A. Intracellular mechanisms of antigen cross presentation in dendritic cells. Curr. Opin. Immunol. 22, 109-117 (2010).

37 Belizaire R, Unanue ER. Targeting proteins to distinct subcellular compartments reveals unique requirements for $\mathrm{MHC}$ class I and II presentation. Proc. Natl Acad. Sci. USA 106, 17463-17468 (2009).

38 Markov OO, Mironova NL, Maslov MA et al. Novel cationic liposomes provide highly efficient delivery of DNA and RNA into dendritic cell progenitors and their immature offsets. J. Control. Release 160, 200-210 (2012).

39 Jilek S, Merkle HP, Walter E. DNA-loaded biodegradable microparticles as vaccine delivery systems and their interaction with dendritic cells. Adv. Drug Deliv. Rev. 57, 377-390 (2005).

40 Ji W, Panus D, Palumbo RN, Tang R, Wang C. Poly(2-aminoethyl methacrylate) with well-defined chain length for DNA vaccine delivery to dendritic cells. Biomacromolecules 12, 4373-4385 (2011).

41 Sun X, Chen S, Han J, Zhang Z. Mannosylated biodegradable polyethyleneimine for targeted DNA delivery to dendritic cells. Int. J. Nanomed. 7 , 2929-2942 (2012).

42 Melhem NM, Gleason SM, Liu XD, Barratt-Boyes SM. High-level antigen expression and sustained antigen presentation in dendritic cells nucleofected with wild-type viral mRNA but not DNA. Clin. Vaccine Immunol. 15, 1337-1344 (2008).

43 Wykes M, Pombo A, Jenkins C, MacPherson GG. Dendritic cells interact directly with naive $\mathrm{B}$-lymphocytes to transfer antigen and initiate class switching in a primary T-dependent response. J. Immunol. 161, 1313-1319 (1998).

44 Le Roux D, Le Bon A, Dumas A et al. Antigen stored in dendritic cells after macropinocytosis is released unprocessed from late endosomes to target B cells. Blood 119, 95-105 (2011).

45 Katas H, Alpar HO. Development and characterisation of chitosan nanoparticles for siRNA delivery. J. Control. Release 115, 216-225 (2006).

46 Gao W, Xiao Z, Radovic-Moreno A, Shi J, Langer R, Farokzad OC. Progress in siRNA delivery using multifunctional nanoparticles. Methods Mol. Biol. 629, 53-67 (2010).

47 Tezuka H, Ohteki T. Regulation of intestinal homeostasis by dendritic cells. Immunol. Rev. 234, 247-258 (2010).

48 Nestle FO, Di Meglio P, Qin J-Z, Nickoloff BJ. Skin immune sentinels in health and disease. Nat. Rev. Immunol. 9, 679-691 (2009).

49 Romani N, Clausen BE, Stoitzner P. Langerhans cells and more: langerinexpressing dendritic cell subsets in the skin. Immunol. Rev. 234, 120-141 (2010).

50 Kaplan DH. In vivo function of Langerhans cells and dermal dendritic cells. Trends Immunol. 31, 446-451 (2010).

51 Watts C, West MA, Zaru R. TLR signalling regulated antigen presentation in dendritic cells. Curr. Opin. Immunol. 22, 124-130 (2010).
52 Dam TK, Brewer CF. Lectins as pattern recognition molecules: the effects of epitope density in innate immunity. Glycobiology 20, 270-279 (2009).

53 Combadiere B, Liard C. Transcutaneous and intradermal vaccination. Hum. Vaccines 7 , 811-827 (2011).

54 Ueno H, Klechevsky E, Schmitt N et al. Targeting human dendritic cell subsets for improved vaccines. Semin. Immunol. 23, 21-27 (2011).

55 Caminschi I, Shortman K. Boosting antibody responses by targeting antigens to dendritic cells. Trends Immunol. 33, 71-77 (2011).

56 Romani N, Thurnher M, Idoyaga J, Steinman RM, Flacher V. targeting of antigens to skin dendritic cells: possibilities to enhance vaccine efficacy. Immunol. Cell Biol. 88, 424-430 (2010).

57 Stoitzner P, Green LK, Jung JY et al. Tumor immunotherapy by epicutaneous immunization requires Langerhans cells. J. Immunol. 180, 1991-1998 (2008).

58 Garcia-Gaumont C, Seksek O, Grzybowska J, Borowski E, Bolard J. delivery systems for antisense oligonucleotides. Pharmacol. Ther. 87, 255-277 (2000)

59 Mahato RI, Takakura Y, Hashida M. Development of targeted delivery systems for nucleic acid drugs. J. Drug Target. 4, 337-357 (1996).

60 Henriksen-Lacey M, Korsholm KS, Andersen P, Perrie Y, Christensen D. Liposomal vaccine delivery systems. Exp. Opin. Drug Del. 8, 505-519 (2011).

61 Wasungu L, Hoekstra D. Cationic lipids, lipoplexes and intracellular delivery of genes. J. Control. Release 116, 255-264 (2006).

62 Landesman-Milo D, Peer D. Altering the immune response with lipid-based nanoparticles. J. Control. Release 161, 600-608 (2012).

63 Li W, Szoka FC. Lipid-based nanoparticles for nucleic acid delivery. Pharm. Res. 24, 438-449 (2007).

64 Slütter B, Bal SM, Ding Z, Jiskoot W, Bouwstra JA. Adjuvant effect of cationic liposomes and $\mathrm{CpG}$ depends on administration route. J. Control. Release 154, 123-130 (2011).

65 Hassane FS, Saleh AF, Abes R, Gait MJ, Lebleu B. Cell penetrating peptides: overview and applications to the delivery of oligonucleotides. Cell. Mol. Life Sci. 67, 715-726 (2010).

66 Vazquez-Calvo A, Saiz J-C, McCullough KC, Sobrino F, Martin-Acebes MA. Acid-dependent viral entry. Virus Res. 167(2), 125-137 (2012). 
67 Mueller J, Kretzschmar I, Volkmer R, Boisguerin P. Comparison of cellular uptake using 22 CPPs in 4 different cell lines. Bioconjug. Chem. 19, 2363-2374 (2008).

68 Lundin P, Johansson H, Guterstam P et al. Distinct uptake routes of cell-penetratng peptide conjugates. Bioconjug. Chem. 19, 2535-2542 (2008).

69 Zaro JL, Shen W-C. Quantitative comparison of membrane transduction and endocytosis of oligopeptides. Biochem. Biophys. Res. Commun. 307, 241-247 (2003).

70 Tünnemann G, Martin RM, Haupt S, Patsch C, Edenhofer F, Cardoso C. Cargodependent mode of uptake and bioavailability of TAT-containing proteins and peptides in living cells. FASEB J. 20 1775-1784 (2006).

71 Summerton JE. A novel reagent for safe, effective delivery of substances into cells. Ann. NY Acad. Sci. 1058, 62-75 (2005).

72 Boudier A, Aubert-Plouëssel A, Gérardin $\mathrm{C}$ et al. Tripartite siRNA micelles as controlled delivery systems for primary dendritic cells. Drug Dev. Ind. Pharm. 35, 950-958 (2009).

73 Diaz-Moscoso A, Vercauteren D, Rejman $\mathrm{J}$ et al. Insights in cellular uptake mechanisms of pDNA-polycationic amphiphilic cyclodextrin nanoparticles (CDplexes). J. Control. Release 143, 318-325 (2010).

74 Dierendonck M, De Koker S, Vervaet C, Remon JP, De Geest BG. Interaction between polymeric multilayer capsules and immune cells. J. Control. Release 161, 592-599 (2012).

75 Hu Y, Litwin T, Nagaraja AR et al. Cytosolic delivery of membrane-impermeable molecules in dendritic cells using $\mathrm{pH}$-responsive core-shell nanoparticles. Nano Lett. 7 , 3056-3064 (2007).

$76 \mathrm{Hu}$ Y, Lu JJ, Atukorale PU et al. Cytosolic delivery mediated via electrostatic surface binding of protein, virus or siRNA cargoes to $\mathrm{pH}$-responsive core-shell gel.

Biomacromolecules 10, 756-765 (2009).

77 Howard KA. Delivery of RNA interference therapeutics using polycation-based nanoparticles. Adv. Drug. Del. Rev. 61, 710-720 (2009).

78 Laga R, Carlisle R, Tangney M, Ulbrich K, Seymour LW. Polymer coatings for delivery of nucleic acid therapeutics. J. Control. Rel. 161, 537-553 (2012).

79 Sharma R, Ghasparian A, Robinson JA, McCullough KC. Synthetic virus-like particles target dendritic cell lipid rafts for rapid endocytosis primarily but not exclusively by macropinocytosis. PLoS ONE e43248 (2012).
80 Midoux P, Pichon C, Yaouanc J-J, Jaffrès P-A. Chemical vectors for gene delivery: a current review on polymers, peptides and lipids containing histidine or imidazole as nucleic acids carriers. Br. J. Pharmacol. 157 , 166-178 (2009).

81 Varkouhi AK, Scholte M, Storm G, Haisma HJ. Endosomal escape pathways for delivery of biologicals. J. Control. Release 151, 220-228 (2011).

82 Gonzalez-Rodriguez ML, Rabasco AM. Charged liposomes as carriers to enhance the permeation through the skin. Exp. Opin. Drug Del. 8, 857-871 (2011).

83 Jiang H-L, Kim TH, Kim Y-K, Park I-Y, Cho M-H, Cho CS. Efficient gene delivery using chitosan-polyethylenimine hybrid systems. Biomed. Mater. 3(2), 025013 (2008).

84 Won Y-W, Lim KS, Kim Y-H. Intracellular organelle-targeted non-viral gene delivery systems. J. Control. Release 152, 99-109 (2011).

85 Nakase I, Kobayashi S, Futaki S. Endosomedisruptive peptides for improving cytosolic delivery of bioactive macromolecules. Biopolym. Peptide Sci. 94, 763-770 (2010).

86 Hafez IM, Maurer N, Cullis PR. On the mechanism whereby cationic lipids promote intracellular delivery of polynucleic acids. Gene Ther. 8, 1188-1196 (2001)

87 Tavernier G, Andries O, Demeester J, Sanders NN, De Smedt SC, Rejman J. mRNA as gene therapeutic: how to control protein expression. J. Control. Release 150, 238-247 (2011).

88 Ceppi M, Ruggli N, Tache V, Gerber H, McCullough KC, Summerfield A. Doublestranded secondary structures on mRNA induce type 1 interferon (IFN a/b) production and maturation of mRNAtransfected monocyte-derived dendritic cells. J. Gene Med. 7, 452-465 (2005).

89 Liu MA. Gene-based vaccines: recent developments. Curr. Opin. Mol. Therapy 12, 86-93 (2010).

90 Dow SW. Liposome-nucleic acid immunotherapeutics. Exp. Opin. Drug Del. 5, 11-24 (2008).

91 Basha G, Novobrantseva TI, Rosin N et al. Influence of cationic lipid composition on gene silencing properties of lipid nanoparticle formulations of siRNA in antigen-presenting cells. Am. Soc. Gene Cell Ther. 19, 2186-2200 (2011).

92 Tesz GJ, Aouadi M, Prot M et al. Glucan particles for selective delivery of siRNA to phagocytic cells in mice. Biochem. J. 436, 351-362 (2011).
93 Subramanya S, Kim S-S, Abraham S et al. Targeted delivery of small interfering RNA to human dendritic cells to suppress Dengue Virus infection and associated proinflammatory cytokine production. J. Virol. 84, 2490-2501 (2010).

94 Akita H, Kogure K, Moriguchi R et al. nanoparticles for ex vivo siRNA delivery to dendritic cells for cancer vaccines: programmed endosomal escape and dissociation. J. Control. Release 143, 311-317 (2010).

95 Kong Y, Ruan L, Ma L, Cui Y, Wang JM, Le Y. RNA interference as a novel and powerful tool in immunopharmacological research. Int. Immunopharm. 7, 417-426 (2007).

96 Davidson BL, McCray PB. Current prospects for RNA interference-based therapies. Nat. Rev. Gen. 12, 329-340 (2011).

97 Akinc A, Zumbuehl A, Goldberg M et al. A combinatorial library of lipid-like materials for delivery of RNAi therapeutics. Nat. Biotechnol. 26, 561-569 (2008).

98 Howard KA, Rahbek UL, Liu X et al. RNA interference in vitro and in vivo using a Chitosan siRNA nanoparticle system. Mol. Ther. 14, 476-484 (2006).

99 Rahbek UL, Howard KA, Oupicky D et al. Intracellular siRNA and precursor miRNA trafficking using bioresponsive copolypeptides. J. Gene Med. 10, 81-93 (2008).

100 Boudier A, Aubert-Plouëssel A, Mebarek $\mathrm{N}$ et al. Development of tripartite polyion micelles for efficient peptide delivery into dendritic cells without altering their plasticity. J. Control. Release 154, 156-163 (2011).

101 Mockey M, Bourseau E, Chandrashekhar $\mathrm{V}$ et al. mRNA-based cancer vaccine: prevention of B16 melanoma progression and metastasis by systemic injection of MART 1 mRNA histidylated lipopolyplexes. Cancer Gene Ther. 14, 802-814 (2007).

102 Kim H-K, Wei H, Kulkarni A, Pogranichniy $\mathrm{RN}$, Thompson DH. Effective targeted gene delivery to dendritic cells via synergetic interaction of mannosylated lipid with DOPE and BCAT. Biomacromolecules 13, 636-644 (2012).

103 Perche F, Benvegnu T, Berchel M et al. Enhancement of dendritic cells transfection in vivo and of vaccintion against $\mathrm{B} 16 \mathrm{~F} 10$ melanoma with mannosylated histidylated lipopolyplexes loaded with tumor antigen messenger RNA. Nanomedicine 7, 445-453 (2011).

104 Su X, Fricke J, Kavanagh DG, Irvine DJ. In vitro and in vivo mRNA delivery using lipid-enveloped $\mathrm{pH}$-responsive polymer 
nanoparticles. Mol. Pharm. 8, 774-787 (2011).

105 Debus H, Baumhof P, Probst J, Kissel T. delivery of messenger RNA using poly(ethylene imine)-poly(ethylene glycol)-copolymer blends for polyplex formation: biophysical characterization and in vitro transfection properties. J. Control. Release 148, 334-343 (2010).

106 Rejman J, Tavernier G, Bavarsad N, Demeester J, De Smedt SC. mRNA transfection of cervical carcinoma and mesenchymal stem cells mediated by cationic carriers. J. Control. Release 147, 385-391 (2010).

107 Khromykh AA. Replicon-based vectors of positive strand RNA viruses. Curr. Opin. Mol. Therapy. 2, 555-569 (2000).

108 Mandl CW. Flavivirus immunization with capsid-deletion mutants: basics, benefits, and barriers. Viral Immunol. 17, 461-472 (2004).

109 Rayner JO, Dryga SA, Kamrud KI. Alphavirus vectors and vaccination. Rev. Med. Virol. 12, 279-296 (2002).

110 Atkins GJ, Fleeton MN, Sheahan BJ. Therapeutic and prophylactic applications of alphavirus vectors. Exp. Rev. Mol. Med. 10, e33 (2008).

111 Racanelli V, Behrens SE, Aliberti J, Rehermann B. Dendritic cells transfected with cytopathic self-replicating RNA induce crosspriming of CD8 + T cells and antiviral immunity. Immunity 20, 47-58 (2004).

112 Frey CF, Bauhofer O, Ruggli N, Summerfield A, Hofmann MA, Tratschin JD. Classical swine fever virus replicon particles lacking the Erns gene: a potential marker vaccine for intradermal application. Vet. Res. 37, 655-670 (2006).

113 Suter R, Summerfield A, Thomann-Harwood LJ, McCullough KC, Tratschin JD, Ruggli N. Immunogenic and replicative properties of classical swine fever virus replicon particles modified to induce IFN-alpha/beta and carry foreign genes. Vaccine 29, 1491-1503 (2011).

114 Suzuki R, Winkelmann ER, Mason PW. Construction and characterization of a single-cycle chimeric flavivirus vaccine candidate that protects mice against lethal challenge with dengue virus type 2. J. Virol. 83, 1870-1880 (2009).

115 Kucharzik T, Lugering N, Rautenberg K et al. Role of $\mathrm{M}$ cells in intestinal barrier function. Ann. NY Acad. Sci. 915, 171-183 (2000).

116 Kyd JM, Cripps AW. Functional differences between $M$ cells and enterocytes in sampling luminal antigens. Vaccine 26, 6221-6224 (2008).

117 Martin-Latil S, Gnadig NF, Mallet A et al. Transcytosis of HTLV-1 across a tight human epithelial barrier and infection of subepithelial dendritic cells. Blood 120(3), 572-580 (2012)

118 Chieppa M, Rescigno M, Huang AY, Germain RN. Dynamic imaging of dendritic cell extension into the small bowel lumen in response to epithelial cell TLR engagement. J. Exp. Med. 203, 2841-2852 (2006).

119 Lelouard H, Fallet M, de Bovis B, Meresse S, Gorvel JP. Peyer's patch dendritic cells sample antigens by extending dendrites through $\mathrm{M}$ cell-specific transcellular pores. Gastroenterology 142, 592-601 e593 (2012).

120 Rescigno M, Urbano M, Valzasina B et al. Dendritic cells express tight junction proteins and penetrate gut epithelial monolayers to sample bacteria. Nat. Immunol. 2, 361-367 (2001).

121 Vallon-Eberhard A, Landsman L, Yogev N, Verrier B, Jung S. Transepithelial pathogen uptake into the small intestinal lamina propria. J. Immunol. 176, 2465-2469 (2006).

122 Soloff AC, Barratt-Boyes SM. Enemy at the gates: dendritic cells and immunity to mucosal pathogens. Cell Res. 20, 872-885 (2010). 